\title{
AUFSATZ
}

\section{Zwei Seiten der gleichen Medaille? Zum theoretischen und empirischen Zusammenhang zwischen der Regierungsform und der Ausgestaltung von Zweikammersystemen}

\author{
Steffen Kailitz
}

Die Zusammenhänge zwischen Präsidentialismus und Bikameralismus wurden trotz der inzwischen gewaltigen Flut an Beiträgen zur Parlamentarismus-Präsidentialismus-Debatte und der anwachsenden Beschäftigung mit dem Bikameralismus bislang nicht systematisch in den Blick genommen. ${ }^{1}$ Dies soll in diesem Beitrag geschehen. Dabei soll belegt werden, dass es, erstens, einen theoretischen Zusammenhang der institutionellen Konzepte Präsidentialismus und Bikameralismus als Elemente der Gewaltenteilung gibt und, zweitens, dass dieser theoretische Zusammenhang sich noch heute in unterschiedlichen Profilen der Ausgestaltung des Bikameralismus in parlamentarischen und präsidentiellen Demokratien niederschlägt. Als ein Zweikammersystem gilt dabei jedes System, in dem es neben einer vom Volk gewählten Versammlung von Repräsentanten, als Minimalausstattung der Demokratie, noch eine zweite gibt, die an der Gesetzgebung beteiligt ist. ${ }^{2}$

Konkret werden die Beziehungen zwischen (1) der Existenz einer Zweiten Kammer, (2) ihrer Stärke, (3) dem Grad ihrer demokratischen Legitimation und (4) einer eigenständigen Funktion (und funktionalen Legitimation) der Zweiten Kammer (separation of purpose zwischen Erster und Zweiter Kammer) auf der einen Seite und der Regierungsform auf der anderen Seite untersucht. ${ }^{3}$ Dabei erfolgt die Auswertung variablen- und nicht fallorien-

1 Vgl. für einen Überblick zum Stand der Parlamentarismus-Präsidentialismus-Debatte: Robert Elgie, From Linz to Tsebelis: Three Waves of Presidential / Parliamentary Studies?, in: Democratization, 12. Jg. (2005), S. $106-122$.

2 Keine der hier untersuchten Demokratien hatte nach 1945 mehr als zwei Kammern. Historisch betrachtet finden sich aber Ausnahmen, in denen die Kammerzahl deutlich höher war. So gab es in Schweden bis 1866 über vier Parlamentskammern.

3 Beim Vergleich der Zweiten Kammern ließen sich viele weitere Punkte berücksichtigen, zum Beispiel die Zahl der Abgeordneten oder die Amtsdauer. Die Vergleiche solcher Punkte sagen etwas über die Unterschiede zwischen Ersten und Zweiten Kammern aus, aber nichts bis wenig über die Unterschiede und Gemeinsamkeiten der Zweikammersysteme und vor allem über ihren Zusammenhang mit der Regierungsform. So lässt sich etwa für diese Untersuchung nur wenig daraus ableiten, dass Zweiten Kammern durchschnittlich 106 Abgeordnete angehören und diese damit - mit Ausnahme Großbritanniens und Taiwans - weniger Mitglieder haben als Erste Kammern. Vgl. dazu Rein Taagepera / Steven Recchia, The Size of Second Chambers and European Assemblies, in: European Journal of Political Research, 41. Jg. (2002), S. 165 - 185. Einzig im Falle gemeinsamer Sitzungen beider Kammern hat dieser Faktor Einfluss auf das Kräfteverhältnis zwischen Erster und Zweiter Kammer. 
tiert. ${ }^{4}$ Es soll dabei gezeigt werden, dass der Zusammenhang zwischen Regierungsform und Bikameralismus in mancher Hinsicht sogar deutlich stärker ist als der bekannte Zusammenhang zwischen Föderalismus und Bikameralismus.

Die Ergebnisse führen auch dazu, dass einem einflussreichen Irrtum von Arend Lijphart nachdrücklich widersprochen werden muss. Im Sinne Lijpharts ist nämlich eine starke Zweite Kammer ein Element der Konsensusdemokratie, während ein Einkammersystem oder ein stark asymmetrisches Zweikammersystem ein Zeichen für eine Mehrheitsdemokratie ist. ${ }^{5}$ Sowohl die theoretischen Grundlagen des Präsidentialismus als auch die überdurchschnittliche Häufigkeit starker und stark legitimierter Zweiter Kammern stehen daher in einem deutlichen Spannungsverhältnis zur folgenden Ansicht Lijpharts: „Presidentialism spells majoritarianism." 6

\section{Bikameralismus und Präsidentialismus als Elemente der Gewaltenteilung}

Das parlamentarische und das präsidentielle Regierungsmodell der Demokratie unterscheiden sich grundlegend hinsichtlich des Verhältnisses von Parlament und Regierung. Die zuerst entstandene präsidentielle Demokratieform ist dabei das Produkt theoretischer Überlegungen, die die „Federalists"7 in brillanter Form auf den Punkt gebracht haben. Die parlamentarische Demokratie hat sich dagegen Schritt für Schritt über mehrere Jahrhunderte mit dem Aufkommen der Parteien und der Demokratie in Großbritannien herausgebildet. ${ }^{8}$ Daraus erklärt sich, dass sich die lange und ehrwürdige Tradition der Gewaltenteilung weit stärker in der Grundkonzeption des Präsidentialismus spiegelt.

Der Kern dieser Denktradition war im Gefolge von John Locke, dass Exekutive und Legislative voneinander getrennt sein müssten, da es die Macht zu sehr balle, wenn die gleichen Personen, die Gesetze verabschieden, auch über ihre Ausführung entscheiden. ${ }^{9}$ Während die US-Amerikaner ihre Verfassung an diesem Grundsatz der institutionellen Machtstreuung orientierten und als „Verfassungspatrioten“ anschließend die Politik an der

4 Aus Platzgründen kann keine ausführlichere Erläuterung der Zuordnung der Länder(jahre) zu bestimmten Kategorien erfolgen. Vgl. hierzu Steffen Kailitz, Parlamentarische, semipräsidentielle und präsidentielle Demokratien im Vergleich. Strukturen und Konsequenzen der Regierungsformen, Habilitationsschrift, Chemnitz 2004.

5 Vgl. Arend Lijphart, Patterns of Democracy. Government Forms and Performance in Thirty-Six Countries, Yale 1999, S. $200-215$.

6 Arend Lijphart, Presidentialism and Majoritarian Democracy: Theoretical Observations, in: Juan Linz / Arturo Valenzuela (Hrsg.), The Failure of Presidential Democracy, Baltimore / London 1994, S. 101. Diese Ansicht teilt Juan Linz, Presidential or Parliamentary Democracy: Does it Make a Difference?, in: ebenda, S. 19.

7 Vgl. Alexander Hamilton / James Madison / John Jay, The Federalist Papers (1787), hrsg. von Pennsylvania State University (zu finden unter: http://www2.hn.psu.edu/faculty/jmanis/ jimspdf. htm, Stand: 20. März 2006).

8 Vgl. Klaus von Beyme, Die parlamentarischen Regierungssysteme in Europa, 2. Auflage, München 1973.

9 Vgl. John Locke, Concerning Civil Government, Second Essay: An Essay Concerning the True Original Extent and End of Civil Government (1690), hrsg. von Pennsylvania State University, S. 82 (zu finden unter: http://www2.hn.psu.edu/faculty/jmanis/locke/civilgo2.pdf, Stand: 12. Mai 2007). 
Theorie ausrichteten, folgte in Großbritannien erst aus der Beobachtung der Praxis der Politik nach und nach die Erkenntnis, dass sich hier ein theoretisch überraschend geschlossenes Modell einer parlamentarischen Regierungsweise herausgebildet hatte, das sich dem US-Modell der präsidentiellen Regierungsform gegenüberstellen lässt. Dabei ist die in Großbritannien entstandene Urvariante der parlamentarischen Demokratie durch den entschiedenen Willen, die Macht bei der Mannschaft aus Regierung und Parlamentsmehrheit zu konzentrieren, zugleich das Modell der Mehrheitsdemokratie. ${ }^{10}$ So hatte Walter Bagehot bereits 1867 die Gewaltenteilung für die politische Praxis Großbritanniens als belanglose Idee abgetan. Die parlamentarische Regierungsform sei nämlich nicht durch eine Gewaltenteilung gekennzeichnet, sondern durch eine nahezu völlige Verschmelzung der exekutiven mit der legislativen Gewalt. Für ihn bedeutete parlamentarische Regierung, dass das Parlament durch seinen „Hauptausschuss“, das Kabinett, selbst den Staat regiere. ${ }^{11}$

Der Bikameralismus lässt sich wie die Gestaltung des Präsidentialismus auf Ideen der Gewaltenteilungstheoretiker zurückführen. In den Schriften der „Federalists“ werden Bikameralismus und Präsidentialismus als gemeinsame Elemente eines Systems der „separation of powers “ untrennbar miteinander verbunden. ${ }^{12}$ Der erste in den „Federalists “ angeführte Grund für eine Teilung der legislativen Gewalt in zwei Kammern lautet: „A senate [...] doubles the security to the people, by requiring the concurrence of two distinct bodies in schemes of usurpation or perfidy, where the ambition or corruption of one would otherwise be sufficient." 13 Der Grundgedanke einer starken Zweiten Kammer ist also, die Gesetzgebung der Volksversammlung zu mäßigen. ${ }^{14}$ Die Rational-Choice-Forschung betont in der Tradition der „Federalists“ bis heute sehr stark, dass eine „Mäßigung“ und damit qualitative Verbesserung der Gesetzgebung dadurch erreicht werde, dass der Bikameralismus eine breitere politische - und damit auch gesellschaftliche - Mehrheit für Entscheidungen erfordere. ${ }^{15}$ Auch die Sichtweise der „Federalists“, dass in heterogenen Gesellschaften ein Zweikammersystem am besten gewährleisten könne, dass es nicht zu einer „Tyrannei der Mehrheit" kommt, herrscht bis heute unter Rational-Choice-Theoretikern vor. ${ }^{16}$

Der zweite wesentliche Grund der „Federalists“, einen starken Bikameralismus zu befürworten, war, dass sie die Politikstabilität bewusst erhöhen und Veränderungen erschweren wollten. ${ }^{17}$ Für Walter Bagehot, den ersten Chronisten der parlamentarischen Regierungsform, erschien es dagegen bezeichnenderweise als das Grundübel zweier gleich starker Parlamentskammern, dass jedes der beiden Häuser die Gesetzgebung lahmlegen könne. ${ }^{18}$ Die Ansicht, dass ein Zweikammersystem die Policy-Stabilität deutlich erhöht, hallt bis heute in

10 Vgl. Arend Lijphart, Patterns, a.a.O., S. 9 - 30.

11 Vgl. Walter Bagehot, The English Constitution (1867), Harmondsworth 1963.

12 Vgl. unter anderem Correa M. Walsh, The Political Science of John Adams. A Study in the Theory of Mixed Government and the Bicameral System, New York 1915.

13 Vgl. Alexander Hamilton / James Madison / John Jay, a.a.O., S. 277. Es ist ungeklärt, ob der Autor der Passagen Hamilton oder Madison war.

14 Eine ausgezeichnete Übersicht zur Entstehung und Geschichte des Bikameralismus bietet: George Tsebelis / Jeanette Money, Bicameralism, Cambridge 1997, S. 13 - 72.

15 Vgl. James M. Buchanan / Gordon Tullock, The Calculus of Consent, Ann Arbor 1962.

16 So unter anderem William H. Riker, The Justification of Bicameralism, in: International Political Science Review, 13. Jg. (1992), S. $101-116$.

17 Alexander Hamilton / James Madison / John Jay, a.a.O., S. 179.

18 Vgl. Walter Bagehot, a.a.O., S. 73. 
der Vetospieler- und Vetopunktforschung nach. Wertneutral lässt sich dabei formulieren, dass eine starke Kammer die Macht des Agendasetzers - und dies ist in den modernen Demokratien die Regierung - begrenzt. ${ }^{19}$ Dies kann einen „Reformstau“ auslösen, aber auch die Gesellschaft polarisierende oder gar offenkundig unsinnige Gesetze verhindern. Eine starke Zweite Kammer verlangsamt gewöhnlich die Gesetzgebung, macht sie aber beständiger.

\section{Unterscheidung der Regierungsformen}

Die Regierungsformen werden hier entlang zweier Achsen unterschieden: auf der ersten nach dem Verhältnis von Regierung und Parlament. In parlamentarischen Demokratien gibt es, erstens, nur eine parlamentarische Legitimationsschiene, und, zweitens, lässt sich die Regierung als Hauptausschuss des Parlaments interpretieren, den die Vollversammlung der Abgeordneten jederzeit abberufen kann. In präsidentiellen Demokratien gibt es dagegen, erstens, getrennte Legitimationsschienen von Parlament und Regierung, und, zweitens, ist die Regierung nicht vom Parlament abberufbar.

Auf der zweiten Achse werden die Regierungsformen nach der Verteilung der Regierungsmacht geordnet, und dabei wird zwischen den unipolaren Exekutivtypen Parlamentarismus und Präsidentialismus einerseits, dem bipolaren Exekutivtyp andererseits unterschieden. Auf dieser Achse ist je nach der Verteilung der Regierungsmacht zwischen tatsächlichen bipolaren Exekutiven und solchen zu unterscheiden, in denen ein Pol klar dominiert. In quasi-parlamentarischen Systemen verteilen sich die Regierungsbefugnisse formal auf die Ämter des Präsidenten und des Premierministers. Das Pendel schlägt aber - unabhängig von den parlamentarischen Mehrheitsverhältnissen - stark zugunsten des Premierministers aus. Er dominiert im innen- und außenpolitischen Bereich. Außerdem ist er nur vom Parlament, nicht vom Präsidenten abhängig. Der Premierminister entscheidet, wen er zum Minister beruft und wen er entlässt. Der Präsident nimmt - wie auch in parlamentarischen Demokratien - faktisch (nahezu) ausschließlich Aufgaben eines Staatsoberhaupts wahr. In Systemen mit dualer Exekutive sind die Regierungsbefugnisse tatsächlich auf die Ämter des Präsidenten und des Premierministers aufgeteilt. Der Präsident ist dabei zugleich Staatsoberhaupt. Die Machtbalance hängt in den Staaten dieses Typs von den parlamentarischen Mehrheitsverhältnissen ab. So lange Präsident und Premierminister der gleichen Partei oder zumindest dem gleichen politischen Lager angehören, gibt es ein Machtgefälle zugunsten des Präsidenten. Gehören der Premierminister und der Präsident unterschiedlichen Parteien an, verschiebt sich die Machtbalance deutlich in Richtung des von der Parlamentsmehrheit abhängigen Premierministers. Wie stark das Pendel ausschlägt, hängt neben den persönlichen Charakteristika des Premierministers davon ab, ob er an der Spitze einer Einpartei- oder einer Koalitionsregierung steht. Die Abgrenzung der Kompetenzen von Präsident und Premierminister in der Verfassung ist in der Regel ausgesprochen vage. Als grobe

19 Vgl. Saul Levmore, Bicameralism: When Are Two Decisions Better than One?, in: International Review of Law and Economics, 12. Jg. (1992), S. 145 - 162; George Tsebelis / Jeanette Money, a.a.O., S. 36 f. Allerdings gingen die „Federalists” von einem politischen System aus, in dem die Nationalversammlung die Agenda bestimmt. 
Grenzlinie lässt sich feststellen, dass der Präsident den außenpolitischen, der Premierminister den innenpolitischen Bereich dominiert. ${ }^{20}$ Diese Trennung wird zwar in Phasen, in denen der Präsident und der Premierminister der gleichen Partei oder zumindest dem gleichen politischen Block angehören, nicht immer eingehalten. Sie markiert aber eine vergleichsweise klare Abgrenzung, wenn Premierminister und Präsident konkurrierenden politischen Lagern angehören. ${ }^{21}$ Dennoch lässt sich durchaus zu folgendem Urteil kommen: Die französische Verfassung - und mit ihr die nach ihrem Vorbild gestalteten Verfassungen mit dualer Exekutive - weichen einer eindeutigen Antwort auf die für jede Verfassung zentrale Frage „Wer regiert?" aus. ${ }^{22}$

In quasi-präsidentiellen Demokratien schlägt die Waage bei der Verteilung der Regierungsbefugnisse - weitgehend unabhängig von den parlamentarischen Mehrheitsverhältnissen - sehr stark zugunsten des Präsidenten aus. Er dominiert den Bereich der Außen- und Innenpolitik. Es gibt in diesen Fällen ein starkes Hierarchiegefälle vom Präsidenten zum Premierminister. Beispiele für diesen Typ sind etwa Russland und die Ukraine.

Diese Modifikation in der Typologie der Regierungssysteme gegenüber der geläufigeren Dreiteilung in Parlamentarismus, Semi-Präsidentialismus und Präsidentialismus erscheint aus folgendem Grund notwendig: Die quasi-parlamentarischen Demokratien wie Island und Irland gleichen den parlamentarischen Demokratien, die quasi-präsidentiellen Demokratien wie Peru und Russland dagegen den präsidentiellen Demokratien. Die Modifikation wurzelt in der Erkenntnis, dass der Typ des Semi-Präsidentialismus zu grob ist. ${ }^{23}$ Die in Russland, Frankreich und Österreich praktizierten Regierungsformen sind derart unterschiedlich, dass sie nicht als Angehörige der gleichen Regierungsformenkategorie behandelt werden sollten. ${ }^{24}$

\section{Unterscheidung in Ein-und Zweikammersysteme}

In jeder repräsentativen Demokratie muss es eine durch das Volk gewählte Versammlung von Repräsentanten geben. ${ }^{25}$ Die Parlamente der Demokratien lassen sich aber danach un-

20 Franzosen leiten aus der Bezeichnung des Präsidenten als „Garant der nationalen Unabhängigkeit, der Integrität des Staatsgebietes und der Einhaltung der Verträge“ (Art. 5 Verf. Frankreichs) eine alleinige Zuständigkeit des Präsidenten für die Außen- und Sicherheitspolitik ab. Für diese Interpretation spricht die in Artikel 52 verankerte Zuständigkeit des Präsidenten für die Aushandlung aller internationalen Verträge. Die Formulierung des Art. 20, die Regierung bestimme die Politik der Nation, legen die Franzosen wiederum so aus, dass diese für die Innenpolitik zuständig sei. Vgl. unter anderem Udo Kempf, Das politische System Frankreichs, 4. Auflage, Wiesbaden 2004, S. $66 \mathrm{f}$.

21 Vgl. Robert Elgie, France, in: ders. (Hrsg.), Semi-Presidentialism in Europe, Oxford 1999, S. 67 -85 .

22 Vgl. dazu ausführlicher Vincent Wright, The Government and Politics of France, 3. Auflage, London 1989, S. 12.

23 Dies ergab sich im Rahmen der Arbeit an meiner Habilitationsschrift, vgl. Steffen Kailitz, a.a.O.

24 Dies spiegelt sich auch in den Arbeiten von Robert Elgie wieder, der aber am Oberbegriff des Semi-Präsidentialismus festhält: Robert Elgie / Iain McMenamin, Divided Executives and Democratisation, Dublin 2006.

25 Dies bedeutet aber keineswegs umgekehrt, dass jeder Staat eine Demokratie ist, in dem es eine vom Volk gewählte Versammlung gibt. Die Liste der Beispiele von Volksdemokratien wie der 
terscheiden, ob es nur diese Versammlung gibt oder noch eine zweite, die an der Gesetzgebung beteiligt, aber nicht die allgemeine Volksvertretung ist. ${ }^{26}$ Diese Definition sagt in erster Linie, was Zweite Kammern nicht sind. Eine positive Definition ist nicht möglich, weil Zweite Kammern sehr unterschiedliche spezifische Funktionen erfüllen und verschiedenartig rekrutiert werden. ${ }^{27}$ Gewöhnlich lässt sich leicht entscheiden, ob ein Parlament eine oder zwei Kammern besitzt. Es gibt allerdings einige Ausnahmen. In Norwegen wählt das Volk ein Parlament. Erst nach der Wahl spaltet sich dieses in zwei Kammern auf. Alle Parlamentarier bestimmen ein Viertel der Abgeordneten aus ihrer Mitte zu Mitgliedern der Zweiten Kammer. Bei besonderen Entscheidungen wie etwa über den Haushalt wird die Trennung zeitweilig aufgehoben, und alle Abgeordneten stimmen gemeinsam ab. Für Island galt bis 1991 das gleiche Prinzip. Der einzige Unterschied war, dass ein Drittel der vom Volk gewählten Abgeordneten der Zweiten Kammer angehörte. Taiwan beschloss im April 2000 einen Verfassungszusatz, der die Wahl zur Zweiten Kammer abschaffte und eine Ernennung gemäß der Sitzverteilung der Ersten Kammer vorsah. Faktisch handelt es sich wie in Norwegen um ein gewähltes Parlament, das sich nach der Wahl in zwei Kammern teilt. ${ }^{28}$ Diese Sonderfälle zwischen Einkammer- und Zweikammersystemen werden im Folgenden Aus-Eins-mach-Zwei-Systeme genannt.

In Botswana, Fidschi und Luxemburg gibt es Organe, die sich auf den ersten Blick als Zweite oder gar Dritte Kammern interpretieren lassen, aber keine sind. Dies ist auf Fidschi und in Botswana das Haus der Stammesführer und in Luxemburg der Staatsrat. Das Haus der Stammesführer ist in den Verfassungen Botswanas und Fidschis nicht erwähnt, und es greift fast ausschließlich bei Traditionsfragen in die Politik ein. Der Luxemburger Staatsrat ist eine außerparlamentarische Kontrollinstitution für Gesetzgebungsverfahren, keine Zweite Kammer. ${ }^{29}$

Ein weiteres Problem kann sich bei der Klassifizierung aus der Frage ergeben, welche von zwei Parlamentskammern die Erste und welche die Zweite ist. So ist in Kolumbien, Paraguay und Uruguay der Senat eher als Erste Kammer und das Abgeordnetenhaus als Zweite Kammer anzusehen. Während beispielsweise die Kolumbianer die 100 Senatoren in einem nationalen Wahlkreis wählen, bestimmen sie bei den Wahlen zum Abgeordnetenhaus in den 23 Departments, den neun nationalen Territorien und der Hauptstadt Bogotá je zwei Abgeordnete. Die Verteilung der restlichen Sitze orientiert sich an der Einwohnerzahl der Gebietseinheiten. Die Wahl der Abgeordneten entspricht in Kolumbien, Paraguay und Uruguay dem gängigen System, mit dem andere lateinamerikanische Länder ihre Se-

DDR bis hin zu den elektoralen Autokratien der Gegenwart (etwa in den zentralasiatischen Staaten) ist lang.

26 So Hans Albrecht Schwarz-Liebermann von Wahlendorf, Struktur und Funktion der so genannten Zweiten Kammer. Eine Studie zum Problem der Gewaltenteilung, Tübingen 1958, S. V. Die Benennung ist unwichtig. In den Niederlanden wird die Erste Kammer als Zweite bezeichnet, die nach dieser Definition Zweite Kammer dagegen als Erste.

27 Vgl. unter anderem Jean Mastias / Jean Grangé, Les secondes chambres du parlament en Europe occidentale, Paris 1987, S. 31.

28 Vgl. Marianne Rinza, Taiwan (Republic of China), in: Dieter Nohlen / Florian Grotz / Christof Hartmann (Hrsg.), Elections in Asia, Bd. 2: South East Asia, East Asia, and the South Pacific, Oxford 2001, S. 528.

29 Vgl. Michael Schroen, Das politische System Luxemburgs, in: Wolfgang Ismayr (Hrsg.), Die politischen Systeme Westeuropas, 2. Auflage, Opladen 1999, S. 387 f. 
natoren bestimmen. ${ }^{30}$ Über die Frage, welche der Kammern in diesen drei Ländern als Erste und welche als Zweite anzusehen ist, besteht Diskussionsbedarf. Für diese Untersuchung genügt folgende Feststellung: In diesen drei Staaten gibt es eine Kammer, um die Nation in ihrer Gesamtheit zu vertreten, und eine weitere, die eher regionale oder lokale Interessen repräsentieren soll. ${ }^{31}$ Die Einordnungsproblematik hat keine Konsequenzen für die Einschätzung der Stärke und des Grads der demokratischen Legitimation. Beide Kammern sind in den drei Staaten direktdemokratisch legitimiert, und in allen drei Fällen - unabhängig davon, welche Kammer als Erste und welche als Zweite gilt - handelt es sich um symmetrische Zweikammersysteme. Auch mit Blick auf die funktionale Legitimation ist diese vollständig gegeben, unabhängig davon, welche Kammer als Erste oder Zweite zu werten ist.

\section{Untersuchungszeitraum und Untersuchungsfälle}

Der Untersuchungszeitraum umfasst die Jahre 1945 bis 2007, vom Beginn der „zweiten Welle“ der Demokratisierung bis zur Gegenwart. ${ }^{32}$ Auf der Grundlage einer Minimaldefinition der Demokratie berücksichtigt die Studie auch defekte Demokratien ${ }^{33}$ wie Kolumbien, Sri Lanka und die Türkei. Ausgespart bleiben aus demokratietheoretischen Gründen konstitutionelle Monarchien wie etwa Liechtenstein und Monaco sowie Kleinststaaten von Andorra bis Vanuatu - mit einer Einwohnerzahl unter 200.000 Einwohnern. Die Kleinststaaten werden dabei ausgegrenzt, weil ihre politische Entwicklung eigenen Gesetzen folgt. 34

Die Basis der Untersuchung sind Länderjahre, nicht Länder. Als demokratisch gilt dabei ein Länderjahr, das auf der kombinierten Demokratie- und Autokratieskala des Polity-IVProjekts $^{35}$ (von -10 für eine vollkommene Diktatur bis zu +10 für eine vollkommene Demokratie) einen Wert von mindestens sechs erreicht (vgl. Tabelle 1). Eine Aufstellung über die Demokratien des Jahres 2007, die Existenz einer Zweiten Kammer, ihre Stärke, demokratische Legitimation und eigenständige Funktion findet sich im Anhang.

30 Daher legen zum Beispiel auch Ronald P. Archer und Matthew Søberg Shugart nahe, den kolumbianischen Senat als Erste Kammer, das kolumbianische Abgeordnetenhaus als Zweite Kammer zu verstehen: dies., The Unrealized Potential of Presidential Dominance in Colombia, in: Scott Mainwaring / Matthew Søberg Shugart (Hrsg.), Presidentialism and Democracy in Latin America, Cambridge 1997, S. 156.

31 Vgl. Detlef Nolte / Mariana Llanos, Starker Bikameralismus? Zur Verfassungslage lateinamerikanischer Zweikammersysteme, in: ZParl, 35. Jg. (2004), H. 1, S. 118.

32 Vgl. Samuel P. Huntington, The Third Wave: Democratization in the Late Twentieth Century, Norman 1993, S. 16.

33 Vgl. zu dem Konzept: Aurel Croissant/ Peter Thiery, Defekte Demokratie. Konzept, Operationalisierung und Messung, in: Hans-Joachim Lauth / Gert Pickel / Christian Welzel (Hrsg.), Demokratiemessung. Konzepte und Befunde im internationalen Vergleich, Wiesbaden 2000, S. 89 -111 .

34 Vgl. Robert A. Dahl/ Edward Tufte, Size and Democracy, Stanford 1973.

35 Vgl. Polity IV Project, Political Regime Characteristics and Transitions, 1800 - 2004, http:// www.cidcm.umd.edu/polity/ (Stand: 12. Oktober 2007). 
Tabelle 1: Verteilung der demokratischen Länderjahre auf die Regierungsformen (1945 bis 2007)

\begin{tabular}{|c|c|c|}
\hline parlamentarisch & quasi-parlamentarisch & präsidentiell \\
\hline \multirow{5}{*}{$\begin{array}{l}\text { Albanien (ab 98), Australien, } \\
\text { Bahamas (ab 77), Bangladesch } \\
\text { (ab 91), Barbados (ab 66), } \\
\text { Belgien, Belize (ab 81), Botswana } \\
\text { (ab 65), Dänemark, Deutschland } \\
\text { (ab 49), Estland (ab 91), Fidschi } \\
\text { (70-87; ab 92), Frankreich } \\
\text { (45-59), Griechenland (45-67), } \\
\text { Großbritannien, Indien (ab 52), } \\
\text { Israel (ab 48), Italien (ab 46), } \\
\text { Jamaika (ab 62), Japan (ab 52), } \\
\text { Kanada, Lettland (ab 91), } \\
\text { Luxemburg, Malta (ab 64), } \\
\text { Mauritius (68-71, ab 76), Nepal } \\
\text { (91-2002), Neuseeland (ab 07), } \\
\text { Niederlande, Norwegen, Pakistan } \\
\text { (47-55), Papua-Neuguinea (ab } \\
\text { 75), Salomonen (ab 78), } \\
\text { Schweden, Spanien (ab 78), Sri } \\
\text { Lanka (48-77)a, Südafrika (ab } \\
\text { 94), Südkorea (60-61), Thailand } \\
\text { (ab 92), Trinidad \& Tobago (ab } \\
\text { 62), Tschechien (ab 92), Türkei } \\
\text { (50-60, 61-71, 72-80, ab 83), } \\
\text { Ungarn (ab 90) }\end{array}$} & $\begin{array}{l}\text { Bulgarien (ab 90), Finnland (ab } \\
\text { 2000), Griechenland (ab 86), } \\
\text { Irland (ab 21), Island (ab 44), } \\
\text { Litauen (ab 91), Mazedonien (ab } \\
\text { 91), Österreich (ab 46), Portugal } \\
\text { (ab 80), Slowakei (ab 92), } \\
\text { Slowenien (ab 91) }\end{array}$ & \multirow{5}{*}{$\begin{array}{l}\text { Argentinien (46-56, 58-62, } \\
\text { 63-66, 73-75, ab 83), Benin (ab } \\
\text { 91), Bolivien (52-64, 79-80, ab } \\
\text { 82), Brasilien (46-64, ab 85), } \\
\text { Chile (55-73, ab 90), Costa Rica } \\
\text { (ab 49), Dominikanische } \\
\text { Republik (ab 78), Ecuador } \\
\text { (48-62, 68-70, ab 79), El } \\
\text { Salvador (ab 84), Guatemala } \\
\text { (46-54, 58-63, 66-82, ab 86), } \\
\text { Honduras (57-63, 71-72, ab 81), } \\
\text { Kolumbien (45-53, ab 58), Kuba } \\
\text { (45-51), Malawi (ab 94), Mexiko } \\
\text { (ab 94), Nicaragua (ab 90), } \\
\text { Nigeria (79-82), Panama (49-51, } \\
\text { 52-68, ab 94), Paraguay (ab 93), } \\
\text { Philippinen (46-72, ab 87), } \\
\text { Surinam (88-90, ab 91), Uganda } \\
\text { (80-84), Uruguay (52-70, ab } \\
\text { 85), USA, Venezuela (45-48, ab } \\
\text { 58), Zypern - griechischer Teil } \\
\text { (ab 74) }\end{array}$} \\
\hline & & \\
\hline & $\begin{array}{l}\text { Albanien (91-96), Finnland } \\
\text { (45-2000), Frankreich (ab 59), } \\
\text { Griechenland (74-86), Kap Verde } \\
\text { (ab 92), Moldawien (ab 91), } \\
\text { Mongolei (ab 90), Polen (ab 89), } \\
\text { Portugal (76-80), Rumänien } \\
\text { (ab 90), Taiwan (ab 2001), Ukrai- } \\
\text { ne (ab 2006) }\end{array}$ & \\
\hline & quasi-präsi & \\
\hline & $\begin{array}{l}\text { Armenien (91-95, ab 97), } \\
\text { Guyana (ab 92), Madagaskar (ab } \\
\text { 93), Mali (ab 92), Mozambique } \\
\text { (94-2004), Namibia (ab 90), } \\
\text { Pakistan (72-76, 88-89), Peru } \\
\text { (56-61, 80-92, ab 2001), } \\
\text { Russland (ab 91), Senegal (ab } \\
\text { 91), Sri Lanka (ab 89), Südkorea } \\
\text { (60, ab 88), Taiwan (92-2001), } \\
\text { Ukraine (91-2006) }\end{array}$ & \\
\hline \multicolumn{3}{|c|}{$\begin{array}{l}\text { Die zweistelligen Zahlen stehen für die jeweiligen Jahreszahlen im 20. Jahrhundert. } \\
\text { a Sri Lanka hieß bis } 1972 \text { Ceylon. } \\
\text { b Für die Zeit des Colegiado (1952 bis 1970) nimmt Uruguay wegen der Kollektivpräsidentschaft eine } \\
\text { Sonderstellung unter den präsidentiellen Demokratien ein. } \\
\text { Ouelle: Figene Zusammenstellung. }\end{array}$} \\
\hline
\end{tabular}

\section{Zusammenhang zwischen Regierungsform und Bikameralismus in vier Dimensionen}

\subsection{Die Existenz einer Zweiten Kammer, die Regierungsform und der Föderalismus}

Zwischen der Wahl der Regierungsform und der Entscheidung für ein Ein- oder Zweikammersystem besteht kein Zusammenhang. In parlamentarischen Länderjahren kommen Einkammersysteme ungefähr genauso häufig vor wie in präsidentiellen Länderjahren. Dagegen unterscheiden sich die (parlamentarischen und quasi-parlamentarischen) Westminster- und Nicht-Westminsterdemokratien stark. Auffallend hoch ist im Vergleich zu den parlamentarischen und präsidentiellen Demokratien der Anteil der Einkammersysteme in Regierungssystemen mit dualer Exekutive und in den quasi-präsidentiellen Demokratien (vgl. Tab. 2). 


\begin{tabular}{|l|c|c|c|c|}
\hline Tabelle 2: Regierungsform und Häufigkeit von Zweikammersystemen (1945 bis 2007) \\
\hline Regierungsform & $\begin{array}{c}\text { Einkammer- } \\
\text { system }\end{array}$ & $\begin{array}{c}\text { Aus-Eins-mach- } \\
\text { Zwei-System }\end{array}$ & $\begin{array}{c}\text { Zweikammer- } \\
\text { system }\end{array}$ & $\mathrm{N}$ \\
\hline parlamentarisch & $37,4 \%$ & $3,8 \%$ & $58,8 \%$ & 1637 \\
\hline quasi-parlamentarisch & $43,0 \%$ & $13,9 \%$ & $43,0 \%$ & 330 \\
\hline duale Exekutive & $58,9 \%$ & $2,7 \%$ & $38,4 \%$ & 224 \\
\hline quasi-präsidentiell & $64,3 \%$ & $0,5 \%$ & $35,3 \%$ & 221 \\
\hline präsidentiell & $45,7 \%$ & $0,0 \%$ & $54,3 \%$ & 803 \\
\hline $\begin{array}{l}\text { Westminstermodell- } \\
\text { Dummy }\end{array}$ & $\begin{array}{c}\text { Einkammer- } \\
\text { system }\end{array}$ & $\begin{array}{c}\text { Aus-Eins-mach- } \\
\text { Zwei-System }\end{array}$ & $\begin{array}{c}\text { Zweikammer- } \\
\text { system }\end{array}$ & \\
\hline Westminster & $23,1 \%$ & $0,0 \%$ & $76,9 \%$ & 778 \\
\hline $\begin{array}{l}\text { Nicht-Westminster } \\
\text { 50,1 \% }\end{array}$ & $8,4 \%$ & $41,5 \%$ & 1283 \\
\hline $\begin{array}{l}\text { Leichte Abweichungen von 100 Prozent in den Zeilen können bei dieser und den folgenden Tabellen } \\
\text { durch Rundungen zustande kommen. Beim Westminster-Dummy sind parlamentarische und quasi- } \\
\text { parlamentarische Demokratien berücksichtigt. } \\
\text { Quelle: Eigene Erhebung. }\end{array}$ \\
\hline
\end{tabular}

Nachfolgend wird die Stärke des Zusammenhangs zwischen der Existenz einer Zweiten Kammer und den Faktoren Regierungsform, Präsidentialismus (Dummy-Variable), Föderalismus (Dummy-Variable) und kategorisierte Bevölkerungsgröße geprüft. Genutzt wird dazu Cramers V, ein Zusammenhangsmaß für nominalskalierte Variablen, das Werte zwischen 1 (perfekter Zusammenhang) und 0 (kein Zusammenhang) annehmen kann. Cramers $\mathrm{V}$ ist immer positiv und ermöglicht daher keine Aussage über die Richtung des $\mathrm{Zu}$ sammenhangs. In den Sozialwissenschaften wird nicht selten bereits ein Wert von 0,3 als starker Zusammenhang interpretiert. ${ }^{36}$ Hier soll aber erst ab 0,4 von einem bedeutenden und ab 0,6 von einem starken Zusammenhang gesprochen werden.

Sehr großes Gewicht bei der Entscheidung, ob ein Staat ein Ein- oder Zweikammersystem wählt, sprechen Politikwissenschaftler gewöhnlich der Bevölkerungsgröße und dem Föderalismus zu. Es gilt folgende Faustregel: Je größer und bevölkerungsreicher ein demokratischer Staat, desto wahrscheinlicher ist ein föderalistischer Staatsaufbau, und desto eher hat der Staat zwei Parlamentskammern. ${ }^{37}$ Dies gilt aber nicht für die Westminsterdemokratien. Unter ihnen finden sich viele kleine Länder mit zwei Kammern wie die Bahamas, Barbados, Belize, Irland, Jamaika sowie Trinidad und Tobago. Diese statistische Beziehung zwischen Föderalismus und Bikameralismus ist bedeutend, aber ebenfalls keineswegs perfekt (vgl. Tabelle 3). Dies liegt daran, dass einerseits alle (der wenigen) föderalen Staaten zwei Kammern haben, umgekehrt aber keineswegs alle nicht-föderalen Staaten nur eine. Unter den Westminsterdemokratien dominieren die nicht-föderalen Staaten klar gegenüber den föderalen (Indien, Kanada, Pakistan ${ }^{38}$ ). Dennoch zeigen der Föderalismus und die Bevölkerungsgröße im Unterschied zum Präsidentialismus einen deutlich größeren Zusammenhang mit dem Bikameralismus (vgl. Tabelle 3). Allerdings gab es, bevor die „Federalists“ ein separation-of-powers-Paket aus Präsidentialismus, Bikameralismus und

36 So etwa auch die Orientierungsempfehlung in Uwe W. Gehring / Cornelia Weins, Grundkurs Statistik für Politologen, Wiesbaden 2004, S. 123.

37 So unter anderem Jean Blondel, Comparative Legislatures, Englewood 1973, S. 32 - 35.

38 Im Falle Pakistans sind nur die demokratischen Länderjahre in diese Untersuchung eingeflossen. 
Föderalismus schnürten, keine historische Verknüpfung zwischen der Bildung von Konföderationen / Föderationen einerseits, dem Bikameralismus andererseits. ${ }^{39}$

\begin{tabular}{|c|c|c|c|c|}
\hline & Regierungsform & Präsidentialismus & Föderalismus & $\begin{array}{c}\text { Bevölkerungsgröße } \\
\text { kategorisiert }\end{array}$ \\
\hline $\begin{array}{ll}\text { Existenz } & \text { Cramers V } \\
\text { Zweite } & \\
\text { Kammer } & \end{array}$ &, $161^{* *}$ &, $041^{*}$ &, $465^{* *}$ & ,466** \\
\hline $\mathrm{N}$ & 3250 & 3250 & 3295 & 2443 \\
\hline \multicolumn{5}{|c|}{$\begin{array}{l}\text { ** Die Korrelation ist auf dem Niveau von 0,01 (2-seitig) signifikant. } \\
\text { * Die Korrelation ist auf dem Niveau von 0,05 (2-seitig) signifikant. } \\
\text { Die Variable Regierungsform wurde entsprechend der Ausführungen in Teil } 2 \text { kategorisiert. Bei den Va- } \\
\text { riablen Präsidentialismus und Föderalismus handelt es sich um Dummy-Variablen. Die metrische Varia- } \\
\text { ble Bevölkerungsgröße (in 1.000) wurde für die Zwecke dieser Berechnung in folgende fünf Kategorien } \\
\text { unterteilt: } 1=0-999 ; 2=1000-9999 ; 3=10000-49999 ; 4=50000-99999 ; 5>100000 . \\
\text { Quelle: Eigene Berechnung. }\end{array}$} \\
\hline
\end{tabular}

Der Trend weist eindeutig in Richtung einer Zunahme von Einkammersystemen. 1950 gab es nur in etwas mehr als einem Viertel der 30 Demokratien der Welt ein Einkammersystem (vgl. Tabelle 4), 2007 dagegen bereits in mehr als der Hälfte (der 88 Demokratien; vgl. Tabelle 5). In parlamentarischen Regierungssystemen ist der Trend besonders ausgeprägt. ${ }^{40}$ Neuseeland, Dänemark, Schweden, Island und Thailand schafften ihre Zweiten Kammern ab. Keine parlamentarische Demokratie mit nur einer Kammer richtete dagegen eine zweite ein. Unter den postkommunistischen parlamentarischen Demokratien führten nur Slowenien und Tschechien noch in den 1990er Jahren Zweikammersysteme ein. Ansonsten zeigt sich bei den neuen Demokratien ein klares Übergewicht von Einkammersystemen. Unter quasi-präsidentiellen Demokratien ist ihr Anteil so hoch, weil diese Regierungsform sich erst in den 1990er Jahren verbreitete. Unter den quasi-präsidentiellen Demokratien schaffte zudem Peru 1993 seine Zweite Kammer ab.

Rechnerisch war 1950 der Anteil der Zweikammersysteme unter den parlamentarischen Regierungssystemen noch weit höher als unter den präsidentiellen; inzwischen haben die Anteile sich angeglichen. Die Unterschiede zwischen den beiden Zeitpunkten liegen aber in hohem Maße nicht an Veränderungen in der Verbreitung des Bikameralismus begründet, sondern darin, dass viele lateinamerikanische Staaten mit präsidentieller Exekutive 1950 nicht demokratisch waren. Tatsächlich ist in den präsidentiellen Demokratien der Trend zu Einkammersystemen kaum weniger ausgeprägt als in parlamentarischen. Unter den präsidentiellen Demokratien schloss Venezuela 1999 die Tore seiner Zweiten Kammer. Ecuador unternahm seinen jüngsten Demokratieanlauf ab 1979 ohne Zweite Kammer, obgleich der Bikameralismus tief in der Verfassungsgeschichte verwurzelt war. Auch Nicaragua, das vor 1984 eine präsidentielle Autokratie war, entschloss sich, nachdem es seit 1911 zwei Kammern hatte, diese im Zuge der Demokratisierung abzuschaffen.

39 Siehe ausführlich dazu George Tsebelis / Jeanette Money, a.a.O., S. $31 \mathrm{f}$.

40 Vgl. zu den Umwandlungsprozessen: Lawrence D. Longley / David M. Olson (Hrsg.), Two Into One. The Politics and Processes of National Legislative Change, Boulder 1991. 
Es waren fast nur Zentralstaaten, die ihre Zweiten Kammern auflösten. Die Ausnahme ist Venezuela. Allerdings war dort der Föderalismus, bevor die Zweite Kammer abgeschafft wurde, bereits über Jahrzehnte ausgehöhlt worden. Zu Grabe getragen wurde er, als nicht mehr das Volk die Gouverneure wählte, sondern der Präsident sie ernannte. Auch gegenwärtig diskutieren hautsächlich Zentralstaaten darüber, ob ihre Zweite Kammer nicht überflüssig ist. Bundesstaaten wie Kanada wollen sie demgegenüber reformieren und dafür nutzen, dass die Bundesstaaten ihre Interessen in die nationalen Entscheidungsprozesse einbringen können.

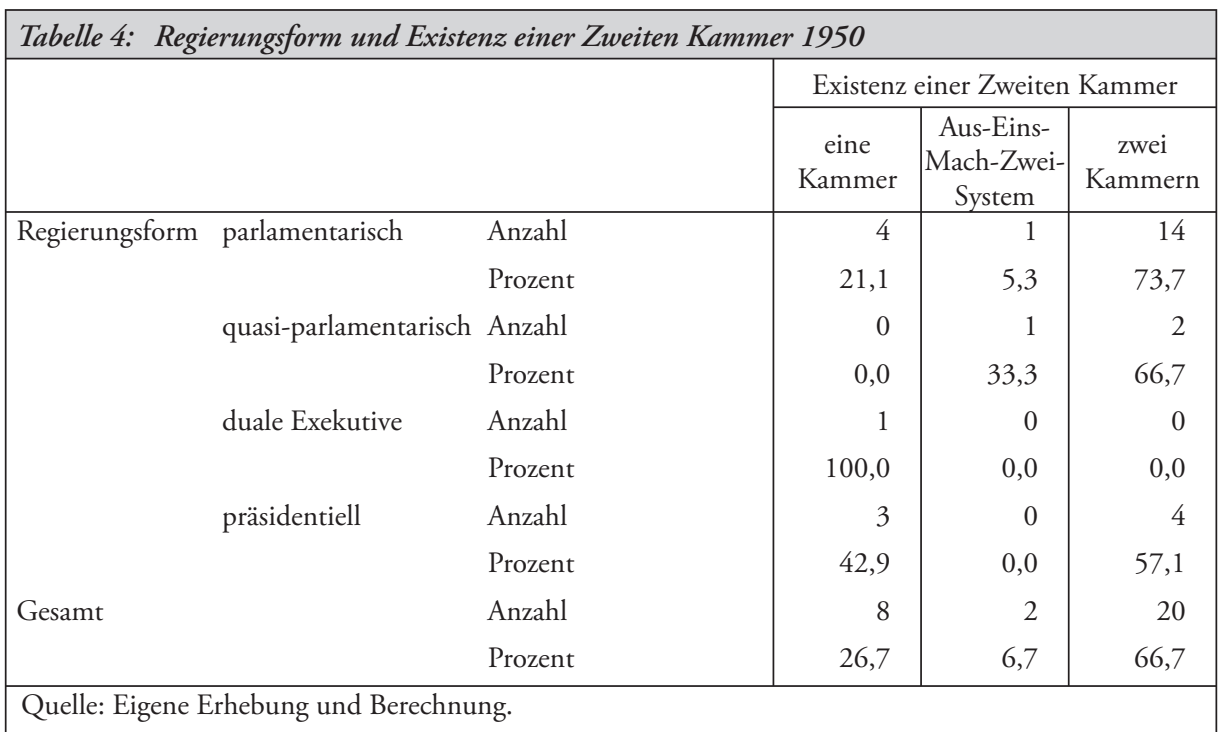

Tabelle 5: Regierungsform und Existenz einer Zweiten Kammer 2007

\begin{tabular}{|c|c|c|c|c|c|}
\hline & & & Existenz & einer Zweiten & Kammer \\
\hline & & & $\begin{array}{c}\text { eine } \\
\text { Kammer }\end{array}$ & \begin{tabular}{|c|} 
Aus-Eins- \\
Mach-Zwei- \\
System
\end{tabular} & $\begin{array}{c}\text { zwei } \\
\text { Kammern }\end{array}$ \\
\hline Regierungsform & parlamentarisch & Anzahl & 16 & 1 & 19 \\
\hline & & Prozent & 44,4 & 2,8 & 52,8 \\
\hline & quasi-parlamentarisch & Anzahl & 8 & 0 & 3 \\
\hline & & Prozent & 72,7 & 0,0 & 27,3 \\
\hline & duale Exekutive & Anzahl & 4 & 1 & 3 \\
\hline & & Prozent & 50,0 & 12,5 & 37,5 \\
\hline & quasi-präsidentiell & Anzahl & 7 & 0 & 3 \\
\hline & & Prozent & 70,0 & 0,0 & 30,0 \\
\hline & präsidentiell & Anzahl & 12 & 0 & 11 \\
\hline & & Prozent & 52,2 & 0,0 & 47,8 \\
\hline Gesamt & & Anzahl & 47 & 2 & 39 \\
\hline & & Prozent & 53,4 & 2,3 & 44,3 \\
\hline
\end{tabular}




\subsection{Die Stärke der Zweiten Kammern}

Bei den folgenden Betrachtungen geht es nur noch um die demokratischen Länderjahre, in denen es Zweite Kammern gab. Mit Blick auf die Stärke von Zweikammersystemen wird zwischen symmetrischen, leicht asymmetrischen und stark asymmetrischen Zweikammersystemen unterschieden. Bei der Einordnung der relativen Stärke der Zweiten Kammer liegt die Verfassungspraxis zugrunde (vgl. Tabelle 6).

\begin{tabular}{|c|c|}
\hline \multicolumn{2}{|l|}{$\begin{array}{l}\text { Tabelle 6: Einordnung der relativen Stärke von Erster und Zweiter Kammer gemäß Verfassungs- } \\
\text { praxis }\end{array}$} \\
\hline & Wert \\
\hline $\begin{array}{l}\text { 1. Symmetrisch sind Zweikammersysteme, in denen beide Kammern über gleiche oder annä- } \\
\text { hernd gleiche Rechte in der Gesetzgebung verfügen. Beispiel: USA. }\end{array}$ & 100 \\
\hline $\begin{array}{l}\text { 2. Asymmetrisch sind Zweikammersysteme, in denen eine der beiden Kammern - in der Pra- } \\
\text { xis durchweg die Erste - politisch größeren Einfluss hat als die andere. In leicht asymme- } \\
\text { trischen Zweikammersystemen ist die Zweite Kammer an den meisten, aber keineswegs an } \\
\text { allen Gesetzgebungsverfahren gleichberechtigt beteiligt. Beispiel: Deutschland. }\end{array}$ & 66,6 \\
\hline $\begin{array}{l}\text { 3. In stark asymmetrischen Zweikammersystemen dominiert die Erste Kammer klar die Ge- } \\
\text { setzgebung. Die Zweite Kammer kann beraten, aber im Kern nicht mitentscheiden. Sie } \\
\text { verfügt nur über ein aufschiebendes Veto. Wenn die Mehrheit der Ersten Kammer gegen } \\
\text { den Willen der Mehrheit der Zweiten Kammer ein Gesetz durchsetzen will, muss sie ledig- } \\
\text { lich ihren Beschluss wiederholen. Beispiel: Großbritannien. }\end{array}$ & 33,3 \\
\hline
\end{tabular}

Stark asymmetrische Zweikammersysteme kommen wegen der Übermacht der Ersten Kammer stets zu Entscheidungen, ohne dass eine Vermittlung zwischen Erster und Zweiter Kammer notwendig ist. In leicht asymmetrischen und symmetrischen Zweikammersystemen sind dagegen Mechanismen notwendig, um den Fall zu regeln, dass sich die Mehrheiten in beiden Kammern uneinig sind. Grundsätzlich gibt es dabei erstens die Möglichkeit eines gemeinsamen Vermittlungsausschusses, zweitens einer gemeinsamen Sitzung beider Häuser und drittens eines Navetteverfahrens, bei dem das Gesetzgebungsprojekt zwischen beiden Kammern hin- und hergleitet, bis beide Kammern zugestimmt haben. Eine gemeinsame Sitzung der Häuser verschiebt im Entscheidungsverfahren gewöhnlich das Gewicht deutlich zugunsten der Ersten Kammer, da diese - außer in Großbritannien - deutlich mitgliederstärker ist als die Zweite. In einem Vermittlungsausschuss sitzen demgegenüber gewöhnlich gleich viele Delegierte aus Erster und Zweiter Kammer. Bei diesem - etwa in den USA genutzten - Verfahren haben beide Kammern das gleiche Gewicht bei der Kompromissbildung. Beim Navetteverfahren ist von grundlegender Bedeutung, wie am Ende die Entscheidung getroffen wird. Drei Varianten stehen für eine Gleichgewichtigkeit von Erster und Zweiter Kammer: (1) es ist kein Ende vorgesehen (zum Beispiel Philippinen); (2) die Kammer, in der das Gesetz zuerst vorgelegt wurde, entscheidet (zum Beispiel Argentinien, Chile); (3) die Kammer, in der die Gesetzesentscheidung der anderen Kammer geprüft wird, entscheidet (zum Beispiel Brasilien, Dominikanische Republik, Mexiko).

Ein symmetrisches Zweikammersystem ist in präsidentiellen Demokratien die Regel, in parlamentarischen und quasi-parlamentarischen Demokratien dagegen die Ausnahme (vgl. 
Tabellen 7 und 8). ${ }^{41}$ Drei von vier parlamentarischen Demokratien mit einem symmetrischen Zweikammersystem (Belgien, Dänemark und Schweden) schafften ihre Zweite Kammer ab. Nur Italien behielt seine starke Zweite Kammer. Westminster- und Nicht-Westminsterdemokratien, also grob gesprochen parlamentarische Mehrheitsdemokratien und solche, die eher der konsensusdemokratischen Richtung zuneigen, unterscheiden sich deutlich. Unter den mehrheitsdemokratischen Westminsterdemokratien finden sich fast ausschließlich stark asymmetrische Zweikammersysteme ${ }^{42}$, in den eher konsensusdemokratischen Nicht-Westminsterdemokratien sind diese dagegen sehr selten. Schwache Zweite Kammern sind also typisch für die mehrheitsdemokratischen Westminsterdemokratien, nicht aber für Nicht-Westminsterdemokratien. Insgesamt sind Zweite Kammern in präsidentiellen Demokratien gewöhnlich weit stärker als in parlamentarischen Demokratien und in Staaten mit dualer Exekutive. Beim Faktor Bikameralismus neigen zudem Länder mit dualer Exekutive weit deutlicher in die Richtung des mehrheitsdemokratischen Westminstermodells als in Richtung eines Konsensusmodells der Demokratie. Dies ist kein Zufall. So zielte die Einführung einer dualen Exekutive in Frankreich darauf, die Regierung gegenüber dem Parlament zu stärken. ${ }^{43}$

\begin{tabular}{|l|c|c|c|c|}
\hline \multicolumn{5}{|c|}{ Tabelle 7: Regierungsform und Stärkeverhältnis Erste und Zweite Kammer (1945 bis 2007) } \\
\hline Regierungsform & $\begin{array}{c}\text { stark } \\
\text { asymmetrisch }\end{array}$ & $\begin{array}{c}\text { leicht } \\
\text { asymmetrisch }\end{array}$ & symmetrisch & $\mathrm{N}$ \\
\hline parlamentarisch & $45,1 \%$ & $39,7 \%$ & $15,2 \%$ & 955 \\
\hline quasi-parlamentarisch & $56,3 \%$ & $43,7 \%$ & $0,0 \%$ & 142 \\
\hline duale Exekutive & $73,9 \%$ & $6,5 \%$ & $19,6 \%$ & 92 \\
\hline quasi-präsidentiell & $20,8 \%$ & $61,1 \%$ & $18,1 \%$ & 72 \\
\hline präsidentiell & $0,0 \%$ & $0,0 \%$ & $100,0 \%$ & 444 \\
\hline $\begin{array}{l}\text { Westminstermodell- } \\
\text { Dummy }\end{array}$ & $\begin{array}{c}\text { stark } \% \\
\text { asymmetrisch }\end{array}$ & $\begin{array}{c}\text { leicht } \\
\text { asymmetrisch }\end{array}$ & symmetrisch & \\
\hline Westminster & $81,3 \%$ & $18,7 \%$ & $0,0 \%$ & 583 \\
\hline Nicht-Westminster & $7,0 \%$ & $62,4 \%$ & $30,6 \%$ & 532 \\
\hline Quelle: Eigene Erhebung und Berechnung. & & & \\
\hline
\end{tabular}

41 In diesem Sinne äußerte sich mit Blick auf Parlamentarismus und Präsidentialismus - allerdings nur beiläufig und ohne die These systematisch geprüft zu haben - zuerst Winfried Steffani, Parlamentarische und präsidentielle Demokratie. Strukturelle Aspekte westlicher Demokratien, Opladen 1979, S. 49. Die Stärke der Zweiten Kammer wurde bei meiner Untersuchung sehr grob gemessen. Für eine ausgefeiltere Messung der Symmetrie Zweiter Kammern vgl. Detlef Nolte I Mariana Llanos, a.a.O., S. 128 - 131. Eine genauere Messung zeigt gewisse Binnenunterschiede zwischen den präsidentiellen Demokratien. So sind etwa die Zweiten Kammern in den USA und Paraguay stärker als in Venezuela und Chile.

42 Nur Australien verfügt über ein leicht asymmetrisches Zweikammersystem. Viele Politikwissenschaftler bezeichnen Australien als Washminster-Demokratie, weil das politische System eine Mischung aus Elementen des britischen Westminstermodells der Demokratie und des Modells der amerikanischen „Föderalisten“ darstellt. Vgl. Christoph M. Haas, Australiens Senat im Washminster-System, in: Gisela Riescher / Sabine Ruß/ Christoph M. Haas (Hrsg.), Zweite Kammern, München / Wien 2000, S. $60-75$.

43 Vgl. Charles de Gaulle, The Bayeux Manifesto, in: Arend Lijphart (Hrsg.), Parliamentary versus Presidential Government, Oxford 1992, S. 139 ff. 


\begin{tabular}{|l|c|c|c|}
\hline \multicolumn{2}{|c|}{ Tabelle 8: Stärke der Zweiten Kammer (1945 bis 2007), Mittelwertvergleich } \\
\hline \multicolumn{1}{|c|}{ Regierungsform } & Mittelwert & $\mathrm{N}$ & Standardabweichung \\
\hline parlamentarisch & 56,6 & 955 & 23,9 \\
quasi-parlamentarisch & 47,8 & 142 & 16,6 \\
duale Exekutive & 48,5 & 92 & 26,8 \\
quasi-präsidentiell & 65,7 & 72 & 20,9 \\
präsidentiell & 100 & 444 & 0 \\
Westminstermodell-Dummy & Mittelwert & $\mathrm{N}$ & Standardabweichung \\
Westminster & 39,5 & 583 & 13 \\
Nicht-Westminster & 74,5 & 532 & 18,9 \\
Föderalismus-Dummy & Mittelwert & $\mathrm{N}$ & Standardabweichung \\
nicht-föderalistisch & 64 & 1058 & 29,3 \\
föderalistisch & 72,4 & 647 & 25,2 \\
\hline Quelle: Eigene Erhebung und Berechnung. \\
\hline
\end{tabular}

\subsection{Demokratische Legitimation Zweiter Kammern und Regierungsform}

Bei den Bestellungsformen Zweiter Kammern werden die in Tabelle 9 aufgeführten Varianten nach dem Grad, in dem sie das Kriterium demokratischer Legitimation erfüllen, unterschieden.

\begin{tabular}{|l|c|}
\hline \multicolumn{2}{|l|}{ Tabelle 9: Varianten der demokratischen Legitimation der Zweiten Kammer } \\
\hline & Wert \\
\hline 1. Das Volk wählt die Mitglieder der Zweiten Kammer. Beispiel: USA. & 100 \\
\hline $\begin{array}{l}\text { 2. Das vom Volk gewählte Parlament wählt die Mitglieder der Zweiten Kammer aus. } \\
\text { Beispiel: Indien. }\end{array}$ & 75 \\
\hline $\begin{array}{l}\text { 3. Der Regierungschef - direkt oder indirekt demokratisch legitimiert - ernennt die } \\
\text { Mitglieder der Zweiten Kammer. Diese erlangen damit eine gewisse demokratische } \\
\text { Legitimation über zwei Banden. Beispiel: Kanada. }\end{array}$ & 50 \\
\hline 4. Die Mitglieder der Zweiten Kammer erben ihre Sitze. Beispiel: Großbritannien vor 1999. & 0 \\
\hline Quelle: Eigene Zusammenstellung. & \\
\hline
\end{tabular}

Häufig werden Mitglieder einer Zweiten Kammer auf unterschiedliche Weise bestellt, so dass es zu Mischformen kommt. Bei der Berechnung der demokratischen Legitimation solcher Zweiter Kammern wurden Mittelwerte gebildet. So erhält ein Zweikammersystem, in dem die Abgeordneten zum Teil ernannt, zum Teil durch demokratisch legitimierte Institutionen gewählt werden, einen Wert von 62,5. Es handelt sich hierbei um das Mittel der Werte für ein Zweikammersystem mit ernannten Mitgliedern (=50) und für ein Zweikammersystem mit zum Teil durch das Parlament bestimmten Mitgliedern (=75). Einem Zweikammersystem mit zum Teil direkt demokratisch legitimierten Mitgliedern und zum Teil indirekt demokratisch legitimierten, wird der Wert 87,5 zugewiesen. Dieser ergibt sich aus dem Mittelwert für Zweikammersysteme mit vom Volk gewählten Mitgliedern (= 100) und für solche mit vom Parlament bestellten Mitgliedern (= 75). 
Die größere Stärke Zweiter Kammern in präsidentiellen Demokratien geht mit einer wesentlich höheren demokratischen Legitimation einher. Die Direktwahl der Mitglieder der Zweiten Kammer ist in präsidentiellen Demokratien die Regel, in parlamentarischen Demokratien eher selten (siehe Tabellen 10 und 11). Dabei gibt es wiederum große Unterschiede zwischen den mehrheitsdemokratischen Westminsterdemokratien und den NichtWestminsterdemokratien. Gegenwärtig kann in elf von zwölf Westminsterdemokratien das Volk nicht darüber (mit)entscheiden, wer in der Zweiten Kammer sitzt. In über der Hälfte der heutigen Nicht-Westminsterdemokratien wählt dagegen das Volk alle oder zumindest einen Teil der Mitglieder der Zweiten Kammer. Bei den Nicht-Westminsterdemokratien zeigt sich somit ein deutlicher Trend in Richtung der Direktwahl.

Typisch für die mehrheitsdemokratischen Westminsterdemokratien, die die Macht bei der Regierung beziehungsweise der Einheit aus Regierung und Parlamentsmehrheit konzentrieren wollen, ist es, die Mitglieder der Zweiten Kammer durch die Regierung zu ernennen (siehe Tabelle 10). Ein anderer Weg ist auch kaum möglich. Eine vom Volk legitimierte starke Zweite Kammer ist dem mehrheitsdemokratischen Westminstermodell wesensfremd. ${ }^{44}$ In den Nicht-Westminsterdemokratien sind überwiegend alle oder ein Teil der Mitglieder Zweiter Kammern indirekt demokratisch legitimiert.

Wenn Mitglieder Zweiter Kammern in Nicht-Westminsterdemokratien territoriale Interessen vertreten sollen, wählt sie gewöhnlich das Parlament in den Gebieten aus, die repräsentiert werden sollen (so zum Beispiel in Südafrika und den Niederlanden). Wenn die subnationalen Parlamente die Zweite Kammer bestellen, hat dies zur Folge, dass sich in dieser das parteipolitische Mehrheitsverhältnis einer Gesamtversammlung der Angehörigen aller Gebietsparlamente spiegelt. ${ }^{45}$ Demgegenüber weist der deutsche Bundesrat eine ganz eigene Bestellungsform auf. Er setzt sich aus Delegierten der Regierungen der deutschen Bundesländer zusammen. ${ }^{46}$ Auch bei dieser Bestellungspraxis herrscht aber das Element der indirekten demokratischen Legitimation vor, weil die Landesparlamente die Ministerpräsidenten wählen.

Gleicht hinsichtlich der demokratischen Legitimation der Mitglieder Zweiter Kammern das Bild der bikameralen parlamentarischen Demokratien einem Flickenteppich, zeigt das der bikameralen präsidentiellen Demokratien ein nahezu einheitliches Webmuster. Nachdem Argentinien 2001 von einer Wahl der Senatoren durch die Provinzparlamente abrückte, wählt in allen präsidentiellen Demokratien das Volk die Mitglieder der Zweiten Kammer. $^{47}$

44 Vgl. Roland Sturm, Zweite Kammern in Westminstermodellen - ein Konstruktionsfehler?, in: André Kaiser (Hrsg.), Regieren in Westminster-Demokratien, Baden-Baden 2000, S. 49.

45 In Österreich wird sogar schlicht der Parteienproporz in den Landtagen zugrunde gelegt, um die 64 Mandate der Zweiten Kammer zu verteilen. Vgl. Franz Fallend, Der Bundesrat in Österreich, in: Gisela Riescher / Sabine Ruß/ Christoph M. Haas (Hrsg.), a.a.O., S. 97 - 113.

46 Daher lässt sich darüber streiten, ob der deutsche Bundesrat überhaupt als ein Parlament gelten kann. So vertritt Klaus von Beyme, Das politische System der Bundesrepublik Deutschland. Eine Einführung, 10. Auflage, Wiesbaden 2004, S. 340, die Ansicht, dass der Bundesrat gar keine Zweite Kammer sei. Der Bundesrat sei nämlich nicht Teil eines Parlaments wie in den Zweikammersystemen Westeuropas.

47 Vgl. mit Blick auf die Zweikammersysteme in den präsidentiellen Demokratien Lateinamerikas: Detlef Nolte / Mariana Llanos, a.a.O. 
Tabelle 10: Regierungsform und demokratische Legitimation Zweiter Kammern (1945 bis 2007)

\begin{tabular}{|l|c|c|c|c|c|c|c|}
\hline Regierungsform & $\begin{array}{c}\text { vererbt/ } \\
\text { ernannt }\end{array}$ & ernannt & $\begin{array}{c}\text { ernannt/ } \\
\text { indirekt }\end{array}$ & indirekt & $\begin{array}{c}\text { indirekt/ } \\
\text { direkt }\end{array}$ & direkt & $\mathrm{N}$ \\
\hline parlamentarisch & $10,0 \%$ & $31,5 \%$ & $1,3 \%$ & $31,1 \%$ & $3,3 \%$ & $22,8 \%$ & 941 \\
\hline quasi-parlamentarisch & $0,0 \%$ & $0,0 \%$ & $56,3 \%$ & $43,7 \%$ & $0,0 \%$ & $0,0 \%$ & 142 \\
\hline duale Exekutive & $0,0 \%$ & $6,5 \%$ & $0,0 \%$ & $53,3 \%$ & $0,0 \%$ & $40,2 \%$ & 92 \\
\hline quasi-präsidentiell & $0,0 \%$ & $19,4 \%$ & $20,8 \%$ & $41,7 \%$ & $0,0 \%$ & $18,1 \%$ & 72 \\
\hline präsidentiell & $0,0 \%$ & $0,0 \%$ & $0,0 \%$ & $4,2 \%$ & $0,0 \%$ & $95,8 \%$ & 429 \\
\hline $\begin{array}{l}\text { Westminstermodell- } \\
\text { Dummy }\end{array}$ & $\begin{array}{l}\text { vererbt/ } \\
\text { ernannt }\end{array}$ & ernannt & $\begin{array}{c}\text { ernannt/ } \\
\text { indirekt }\end{array}$ & indirekt & $\begin{array}{c}\text { indirekt/ } \\
\text { direkt }\end{array}$ & direkt & \\
\hline Westminster & $15,1 \%$ & $50,8 \%$ & $12,9 \%$ & $10,5 \%$ & $0,0 \%$ & $10,8 \%$ & 583 \\
\hline Nicht-Westminster & $1,2 \%$ & $0,0 \%$ & $3,3 \%$ & $56,8 \%$ & $6,0 \%$ & $32,8 \%$ & 518 \\
\hline Quelle: Eigene Berechnung.
\end{tabular}

Zweite Kammern in parlamentarischen Regierungssystemen sind weit schwächer demokratisch legitimiert als in präsidentiellen. Auffallend stark unterscheidet sich die durchschnittliche demokratische Legitimation Zweiter Kammern in präsidentiellen und quasi-präsidentiellen Demokratien. Im Unterschied zum Präsidentialismus sind die quasi-präsidentiellen Demokratien nicht darauf angelegt, die Macht zwischen starken Institutionen zu teilen, sondern wollen die Macht beim Präsidenten konzentrieren (siehe als anschauliches Beispiel Russland). Westminsterdemokratien und Nicht-Westminsterdemokratien unterscheiden sich ebenfalls stark. Zweite Kammern in den ersteren sind gewöhnlich nur schwach oder gar nicht demokratisch legitimiert. Eine weit stärkere demokratische Legitimation können Mitglieder Zweiter Kammern in bikameralen Nicht-Westminsterdemokratien vorweisen.

\begin{tabular}{|l|c|c|c|}
\hline \multicolumn{4}{|c|}{ Tabelle 11: Regierungsform und demokratische Legitimation Zweiter Kammern (1945 bis 2007), } \\
Mittelwertvergleich
\end{tabular}




\subsection{Eigenständige funktionale Legitimation der Zweiten Kammer (separation of purpose) und Regierungsform}

Die Bezeichnung als „Kammer der Berater“48 in Japan drückt deutlich aus, dass die Zweite Kammer in diesem Land keine spezifische Funktion hat. Die einzige Aufgabe und damit auch funktionale Legitimation solcher Zweiter Kammern besteht im Sinne der Gewaltenteilungstheoretiker darin, dass sie die Gesetzgebung der Ersten „abkühlen“. Schon die „Federalists“ betonten aber, dass eine „separation of powers“ ohne eine „separation of purpose“ hohl sei. ${ }^{49}$ Den Zweiten Kammern, die über keine spezifische funktionale Legitimation verfügen, soll dabei keineswegs die Existenzberechtigung abgesprochen werden. Eine „separation of purpose", die das Organ in den Augen der Bevölkerung rechtfertigt, erscheint aber auch dann von Bedeutung, wenn berücksichtigt wird, dass in der politischen Praxis Zweiter Kammern häufig parteipolitische Konfliktlinien dominieren und am Zweck der

\begin{tabular}{|c|c|}
\hline $\begin{array}{l}\text { Tabelle 12: Bewertung der eigenständigen funktionalen Legitimation der Zweiten Kamr } \\
\text { (separation of purpose) }\end{array}$ & \\
\hline & Wert \\
\hline $\begin{array}{l}\text { 1. Die Zweite Kammer dient dazu, subnationale Gliederungen auf zentralstaatlicher Ebene zu } \\
\text { repräsentieren. Beispiele sind die USA und Deutschland. Diese Legitimationsschiene er- } \\
\text { scheint sehr bedeutend, weil sie einem Zerfall des Staatswesens dadurch entgegenwirkt, dass } \\
\text { sich auch die Bevölkerungen kleinerer territorialer Teile des Staates auf zentralstaatlicher Ebe- } \\
\text { ne ausreichend repräsentiert fühlen. Vor allem in ethnisch heterogenen Staaten kann eine so } \\
\text { bestellte Zweite Kammer dazu beitragen, das Konfliktpotential zu senken. In föderalen Staa- } \\
\text { ten sind daher am deutlichsten eine eigenständige Funktion und damit auch eine starke funk- } \\
\text { tionale Legitimation der Zweiten Kammer vorhanden. }\end{array}$ & 100 \\
\hline $\begin{array}{l}\text { 2. Die Zweite Kammer wird teilweise durch das Einbringen der Interessen von Teilgebieten des } \\
\text { Staates auf nationaler Ebene legitimiert. Ein Beispiel ist Belgien. }\end{array}$ & 75 \\
\hline $\begin{array}{l}\text { 3. Die Zweite Kammer soll dazu dienen, die soziale Schichtung der Gesellschaft zu repräsentie- } \\
\text { ren. Ein Beispiel ist Slowenien. In einer modernen Demokratie liegt der Wert für die funktio- } \\
\text { nale Legitimation durch die Repräsentation der sozialen Schichtung unter jener für eine ter- } \\
\text { ritoriale Repräsentation, weil bei dieser Legitimationsvariante das zu repräsentierende Subjekt } \\
\text { wie bei der Ersten Kammer die nationale Bevölkerung ist. Es lässt sich aber mit guten Grün- } \\
\text { den behaupten, dass die Repräsentation „ständische“"Interessen in der modernen Demokra- } \\
\text { tie keine Zweite Kammer mehr erfordert. Bei der Herausbildung der Konfliktlinien der west- } \\
\text { europäischen Parteiensysteme, die das politische Geschehen in den Nationalversammlungen } \\
\text { prägten, spielten nämlich nicht zuletzt grundlegende „,ständische“ Konfliktlinien (Arbeiter vs. } \\
\text { Unternehmer) eine Hauptrolle. }\end{array}$ & 50 \\
\hline $\begin{array}{l}\text { 4. Die Zweite Kammer ist an der Vertretung einer vormodernen Gesellschaftsstruktur ausge- } \\
\text { richtet, die nicht den gegenwärtigen Verhältnissen entspricht. So ist nicht vorgesehen, die } \\
\text { „Stände“, zum Beispiel die Arbeiter, gemäß ihres heutigen Bevölkerungsanteils zu repräsentie- } \\
\text { ren. Ein Beispiel ist Irland. }\end{array}$ & 25 \\
\hline
\end{tabular}

48 Vgl. Beate Rosenzweig, Das japanische Oberhaus - Die Kammer der Berater, in: Gisela Riescher I Sabine Ruß I Christoph M. Haas (Hrsg.), a.a.O., S. 220 - 234.

49 Vgl. Alexander Hamilton / James Madison / John Jay, a.a.O., S. 231. Vgl. zum Verhältnis der „separation of powers” und der „separation of purpose”: Gary W. Cox / Matthew D. McCubbins, Political Structure and Economic Policy: The Institutional Determinants of Policy Outcomes, in: Stephan Haggard / Matthew D. McCubbins (Hrsg.), Presidents, Parliaments and Policy, Cambridge 2001, S. $21-63$. 


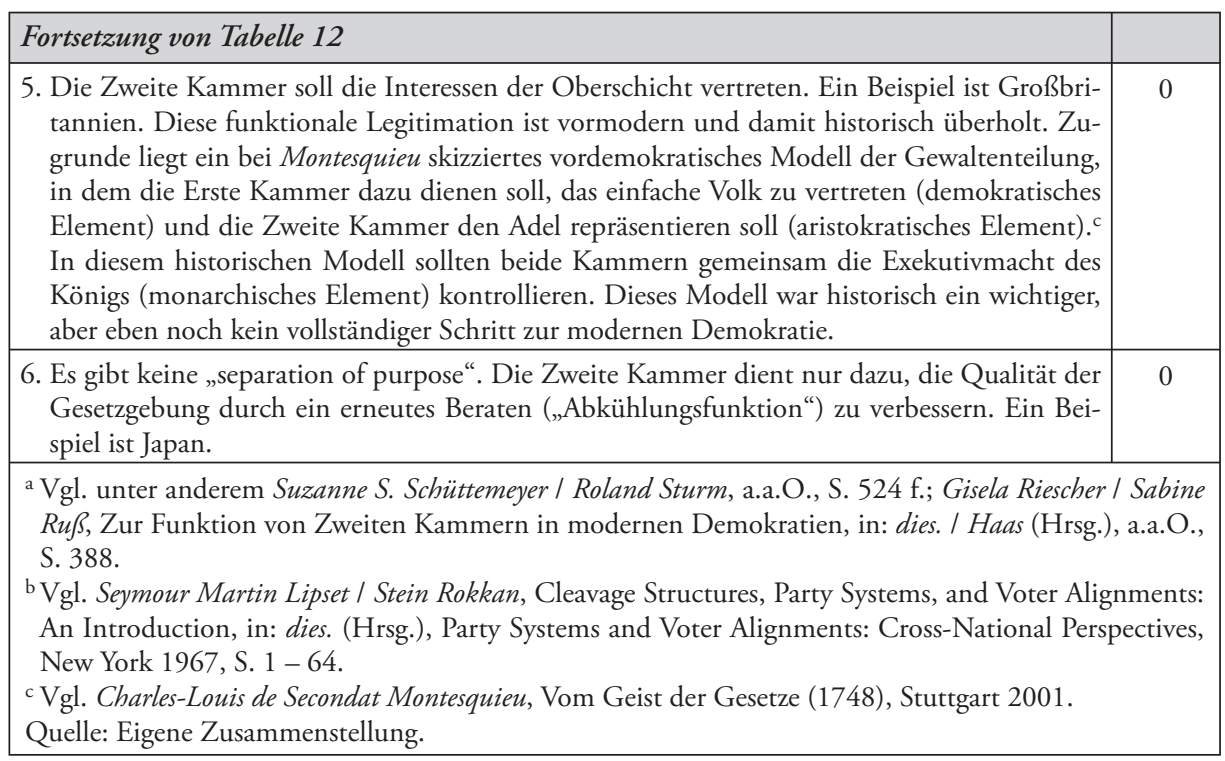

Zweiten Kammer ausgerichtete Konfliktlinien in den Hintergrund treten. ${ }^{50}$ Mit Blick auf die Bewertung der eigenständigen funktionalen Legitimationen Zweiter Kammern werden mit einer normativen Bewertung aus der Perspektive der modernen Demokratietheorie die in Tabelle 12 aufgeführten Varianten unterschieden.

Der Blick auf die demokratischen Länderjahre mit Zweiten Kammern zeigt: In präsidentiellen Demokratien haben Zweite Kammern in der Regel eine starke eigenständige funktionale Legitimation, in parlamentarischen Demokratien gewöhnlich keine oder nur eine schwache (vgl. Tabellen 13 und 14). Besonders häufig fehlt den Zweiten Kammern in Westminsterdemokratien eine die Ansprüche an eine moderne Demokratie befriedigende, eigenständige Funktion. Beispiele sind etwa die fünf kleinsten Westminsterdemokratien (Bahamas, Barbados, Belize, Jamaika sowie Trinidad und Tobago). Zweite Kammern in Nicht-Westminsterdemokratien, die dazu dienen, die Interessen von Teilgebieten auf nationaler Ebene zu vertreten, nehmen diese Aufgabe gewöhnlich auch vollständig wahr, während dies bei Zweiten Kammern in Westminsterdemokratien wie in Kanada häufig nur halbherzig geschieht.

Die Zweiten Kammern in bikameralen präsidentiellen Demokratien werden mit Ausnahme eines Falles, der Philippinen, gegenwärtig durchweg oder zumindest teilweise dadurch legitimiert, dass durch sie territoriale Teilinteressen in die nationale Entscheidungsfindung eingebracht werden sollen (siehe Tabelle 13). Dies geht fast immer mit einer direkten demokratischen Legitimation in den jeweiligen Teilgebieten einher. Dabei folgten die meisten präsidentiellen Demokratien dem „großen Kompromiss“ bei der Gründung der

50 Vgl. Suzanne S. Schüttemeyer / Roland Sturm, Wozu Zweite Kammern? Zur Repräsentation und Funktionalität Zweiter Kammern in westlichen Demokratien, in: ZParl, 23. Jg. (1992), H. 3, S. 528 f.; Adrian Vatter, Politische Institutionen und ihre Leistungsfähigkeit. Der Fall des Bikameralismus im internationalen Vergleich, in: ZParl, 33. Jg. (2002), H. 1, S. 125 - 133. 
USA (so unter anderem Argentinien, Bolivien, Chile und die Dominikanische Republik): Wenn das Volk seine Repräsentanten wählt, gilt „one person, one vote“, im Senat haben dagegen unabhängig von Größe und Bevölkerungszahl die Bundesstaaten die gleiche Stimmzahl - „one state, one vote“51.

Die Überschrift über der Geschichte vieler Zweikammersysteme in parlamentarischen Demokratien $^{52}$ könnte im Unterschied zu den präsidentiellen lauten: eine Institution auf der Suche nach ihrem (verlorenen) Grund. Dies gilt vor allem für die mehrheitsdemokratischen Westminsterdemokratien. Das spezifische Problem Großbritanniens ist, dass hier eine Teilinstitution des historischen Kompromisses zwischen demokratischer (Erste Kammer), aristokratischer (Zweite Kammer) und monarchischer Herrschaftsform (König) überdauert hat, obgleich sie ihren Zweck überlebt hat. Die Briten exportierten zudem die Zweite Kammer in zahlreiche Kolonien, in denen der historische Grund, der in Großbritannien zu einer Einführung von Ober- und Unterhaus geführt hatte, gar nicht vorhanden war. Der Unterschied zwischen den mehrheitsdemokratischen Westminsterdemokratien und den Nicht-Westminsterdemokratien fällt bei der funktionalen Legitimation allerdings deutlich geringer aus als mit Blick auf die Stärke der Zweiten Kammer und ihre demokratische Legitimation.

\begin{tabular}{|c|c|c|c|c|c|c|}
\hline \multicolumn{7}{|c|}{$\begin{array}{l}\text { Tabelle 13: Zusammenhang von Regierungsform und der spezifischen funktionalen Legitimation } \\
\text { Zweiter Kammern (1945 bis 2007) }\end{array}$} \\
\hline Regierungsform & $\begin{array}{l}\text { keine spezifische } \\
\text { Funktion oder } \\
\text { Oberschicht }(0)\end{array}$ & $\begin{array}{l}\text { teilweise } \\
\text { Stände } \\
(25)\end{array}$ & $\begin{array}{l}\text { Stände } \\
(50)\end{array}$ & \begin{tabular}{|c|} 
teilweise \\
Territorien \\
$(75)$ \\
\end{tabular} & $\begin{array}{c}\text { Territorien } \\
(100)\end{array}$ & $\mathrm{N}$ \\
\hline parlamentarisch & $61,5 \%$ & $0,0 \%$ & $6,2 \%$ & $8,1 \%$ & $24,2 \%$ & 941 \\
\hline quasi-parlamentarisch & $0,0 \%$ & $44,4 \%$ & $12,0 \%$ & $0,0 \%$ & $43,7 \%$ & 142 \\
\hline duale Exekutive & $46,7 \%$ & $0,0 \%$ & $0,0 \%$ & $53,3 \%$ & $0,0 \%$ & 92 \\
\hline quasi-präsidentiell & $13,9 \%$ & $0,0 \%$ & $0,0 \%$ & $20,8 \%$ & $65,3 \%$ & 72 \\
\hline präsidentiell & $9,0 \%$ & $0,0 \%$ & $0,0 \%$ & $22,5 \%$ & $68,5 \%$ & 444 \\
\hline $\begin{array}{l}\text { Westminstermodell- } \\
\text { Dummy }\end{array}$ & $\begin{array}{l}\text { keine spezifische } \\
\text { Funktion oder } \\
\text { Oberschicht }(0)\end{array}$ & $\begin{array}{c}\text { teilweise } \\
\text { Stände } \\
(25)\end{array}$ & $\begin{array}{l}\text { Stände } \\
(50)\end{array}$ & $\begin{array}{l}\text { teilweise } \\
\text { Territorien } \\
\quad(75)\end{array}$ & $\begin{array}{l}\text { Territorien } \\
\quad(100)\end{array}$ & $\mathrm{N}$ \\
\hline Westminster & $56,2 \%$ & $11,3 \%$ & $0,0 \%$ & $11,3 \%$ & $21,3 \%$ & 583 \\
\hline Nicht-Westminster & $44,4 \%$ & $0,0 \%$ & $13,4 \%$ & $2,2 \%$ & $40,0 \%$ & 532 \\
\hline
\end{tabular}

51 Vgl. ausführlich zur Entwicklung des Modells: U.S. Senate, Creation of the Senate, Washington 1987.

52 Vgl. dazu auch Werner J. Patzelt, Grundriss einer Morphologie der Parlamente, in: ders. (Hrsg.), Evolutorischer Institutionalismus. Theorie und exemplarische Studien zu Evolution, Institutionalität und Geschichtlichkeit, Würzburg 2007, S. 483 - 564. 


\begin{tabular}{|l|c|c|c|}
\hline \multicolumn{3}{|c|}{ Tabelle 14: Regierungsform und Grad der spezifischen funktionalen Legitimation Zweiter } \\
Kammern, Mittelwertvergleich (1945 bis 2007) \\
\hline Regierungsform & Mittelwert & $\mathrm{N}$ & Standardabweichung \\
\hline parlamentarisch & 33,4 & 941 & 43,8 \\
quasi-parlamentarisch & 60,7 & 142 & 35,5 \\
duale Exekutive & 39,9 & 92 & 37,6 \\
quasi-präsidentiell & 80,9 & 72 & 34,2 \\
präsidentiell & 85,4 & 444 & 28,8 \\
Westminstermodell-Dummy & Mittelwert & $\mathrm{N}$ & Standardabweichung \\
Westminster & 32,1 & 583 & 42,1 \\
Nicht-Westminster & 41,2 & 518 & 44,9 \\
Föderalismus-Dummy & Mittelwert & $\mathrm{N}$ & Standardabweichung \\
nicht-föderalistisch & 26,3 & 1044 & 38,7 \\
föderalistisch & 92,6 & 647 & 15,1 \\
\hline Quelle: Eigene Erhebung und Berechnung. \\
\hline \multicolumn{3}{|l}{} \\
\hline
\end{tabular}

\section{Ergebnisse: starker Zusammenhang zwischen Ausgestaltung des Bikameralismus und Regierungsform}

Bikameralismus wie Präsidentialismus sind „Kinder“ der Gewaltenteilungstradition. Zwar gibt es keinen empirischen Zusammenhang zwischen der Häufigkeit eines Zweikammersystems und der Regierungsform; sehr wohl finden sich aber starke Zusammenhänge zwischen der Ausgestaltung des Zweikammersystems und der Regierungsform. Zweite Kammern sind in präsidentiellen Demokratien gewöhnlich stärker als in parlamentarischen Demokratien. Sie haben zudem deutlich häufiger eine stärkere demokratische Legitimation und eine eigenständige Funktion, nämlich die Vertretung bestimmter Regionen der Nation. Besonders klar treten die Unterschiede zutage, wenn das Westminstermodell als Urform des Parlamentarismus dem Präsidentialismus gegenübergestellt wird. Die australische Kombination einer Westminsterdemokratie mit einer direkt gewählten, föderal ausgerichteten und relativ starken Zweiten Kammer erscheint daher als sehr ungewöhnlich. ${ }^{53}$ Der historische Bezug der Entstehung von Bikameralismus und Präsidentialismus prägt noch erheblich die Ausgestaltung der Demokratieformen. Mit Blick auf die Stärke und die demokratische Legitimation der Zweiten Kammern fällt die Beziehung zwischen Bikameralismus und Präsidentialismus deutlich stärker aus als die zwischen Bikameralismus und Föderalismus. Der Zusammenhang zwischen der Präsidentialismusvariable und der Stärke des Bikameralismus ist mit fast 0,8 ausgesprochen hoch. Nur mit Blick auf die eigenständige Funktion der Zweiten Kammer hat die Föderalismus-Variable einen größeren Einfluss als die DummyVariable Präsidentialismus/Nicht-Präsidentialismus. Dies ist unmittelbar einleuchtend, da die Klassifikation der funktionalen Legitimation selbst dieses Ergebnis präjudiziert. Die mit

53 Vgl. Bruce Ackermann, The Separation of Powers, in: Harvard Law Review, 113. Jg. (2000), S. 634 - 729. Er fällte bei seiner Untersuchung zur Gewaltenteilung folgendes Urteil: „The country's constitution is a fascinating hybrid of British and American elements." (S. 674) 
dem höchsten Wert versehene eigenständige Legitimation ist nämlich die Repräsentation territorialer Interessen auf nationaler Ebene. ${ }^{54}$ Zwischen Föderalismus und Präsidentialismus gibt es dabei einen schwachen Zusammenhang. Präsidentielle Demokratien haben etwas häufiger föderale Strukturen als parlamentarische Demokratien. Ein weiterer Punkt erscheint bedeutsam: Die drei Bikameralismus-Variablen sind keineswegs unabhängig voneinander. Die Stärke der Zweiten Kammer und der Grad ihrer demokratischen Legitimation korrelieren ausgesprochen stark miteinander. Der Zusammenhang zwischen der Stärke und der funktionalen Legitimation ist ebenfalls bedeutsam, aber schwächer ausgeprägt (vgl. Tabelle 15).

\begin{tabular}{|c|c|c|c|c|c|}
\hline \multicolumn{6}{|c|}{ Tabelle 15: Institutionelle Faktoren und die Ausgestaltung des Bikameralismus } \\
\hline & & $\begin{array}{l}\text { Existenz } \\
\text { Zweiter } \\
\text { Kammer }\end{array}$ & Stärke & \begin{tabular}{c|} 
demo- \\
kratische \\
Legitimation
\end{tabular} & $\begin{array}{l}\text { eigen- } \\
\text { ständige } \\
\text { Funktion }\end{array}$ \\
\hline \multirow[t]{2}{*}{ Regierungsform } & &, $16^{* *}$ &, $577^{* *}$ &, $479^{* *}$ &, $453^{* *}$ \\
\hline & $\mathrm{N}$ & 3235 & 1705 & 1676 & 1691 \\
\hline \multirow[t]{2}{*}{ Präsidentialismus } & Cramers V & 034 &, $781^{* *}$ &, $664^{* *}$ &, $46^{* *}$ \\
\hline & $\mathrm{N}$ & 3235 & 1705 & 1676 & 1691 \\
\hline \multirow[t]{2}{*}{ Föderalismus } & Cramers V &, $462^{* *}$ &, $244^{* *}$ &, $415^{* *}$ &, $748^{* *}$ \\
\hline & $\mathrm{N}$ & 3280 & 1734 & 1720 & 1720 \\
\hline \multirow[t]{2}{*}{ Stärke } & Cramers V & ------a & 1 &, $645^{* *}$ &, $387^{* *}$ \\
\hline & $\mathrm{N}$ & ------ & 1720 & 1720 & 1720 \\
\hline \multirow[t]{2}{*}{ demokratische Legitimation } & Cramers V & ----- &, $645^{* *}$ & 1 &, $502^{* *}$ \\
\hline & $\mathrm{N}$ & ------ & 1720 & 1720 & 1720 \\
\hline \multirow[t]{2}{*}{ eigenständige Funktion } & Cramers V & ------ &, $387^{* *}$ &, $502^{* *}$ & 1 \\
\hline & $\mathrm{N}$ & ------ & 1720 & 1720 & 1720 \\
\hline \multicolumn{6}{|c|}{$\begin{array}{l}\text { ** Die Korrelation ist auf dem Niveau von 0,01 (2-seitig) signifikant. } \\
\text { a Eine Berechnung ist nicht möglich, da die Stärke der Zweiten Kammer nur für die Länderjahre mit } \\
\text { Zweikammersystem angegeben wurde. } \\
\text { Quelle: Eigene Berechnung. }\end{array}$} \\
\hline
\end{tabular}

Es ist kein Zufall, dass Zweite Kammern in präsidentiellen Demokratien gewöhnlich stärker sind als in parlamentarischen Demokratien. Nur wenn Regierung und Parlament miteinander verschränkt sind, hat die Antwort auf die Frage, ob es eine mächtige Zweite Kammer gibt, Folgen für die Regierungsstabilität. In einem symmetrischen Zweikammersystem sind nämlich beide Kammern nicht nur gleichberechtigt in der Gesetzgebung, sondern auch beide an den Verfahren der Amtsenthebung eines Regierungschefs beteiligt. In einer präsidentiellen Demokratie ist dies unproblematisch, weil kein Misstrauensvotum gegen die Regierungsspitze möglich ist. Kann aber in einer parlamentarischen Demokratie das Misstrauensvotum von beiden Kammern ausgehen, macht dies das Überleben von Regierungen deutlich schwieriger. Dies gilt besonders dann, wenn die Zweite Kammer erheblich

54 Die sehr deutlichen Zusammenhänge zwischen Föderalismus und Bikameralismus verschwinden allerdings, wenn die Bevölkerungsgröße als Kontrollvariable genutzt wird, während die Zusammenhänge zwischen Regierungsform und Bikameralismus erhalten bleiben. 
anders als die Erste Kammer bestellt wird, denn dann werden unterschiedliche Mehrheitsverhältnisse in beiden Kammern wahrscheinlicher. Tritt dieser Fall ein, fehlt dem Premierminister in einer der beiden Kammern das Vertrauen der Mehrheit und er kann gestürzt werden. Es verwundert daher nicht, dass Italien, die einzige parlamentarische Demokratie, die noch ein symmetrisches Zweikammersystem hat, beide Kammern auf gleiche Weise bestellt. Zudem hat die Zweite Kammer keine eigenständige funktionale Legitimation. Der faktische Unterschied zu einem Aus-Eins-Mach-Zwei-System wie in Norwegen, das einen Bikameralismus nur vorspielt, ist gering. Obgleich es aber in Italien keinerlei „separation of purpose“ der beiden Kammern gibt, war die Regierungsstabilität von 1945 an im Vergleich zu anderen parlamentarischen Demokratien sehr gering. Zwischen Juli 1948 und Dezember 1988 gab es in Italien 48 Regierungen. Die „stabilste“ Regierung überdauerte unter der Führung des Sozialisten Bettino Craxi 1.150 Tage. Die geringe Regierungsstabilität dürfte aber in Besonderheiten des italienischen Parteiensystems, nicht im symmetrischen Zweikammersystem wurzeln. Giovanni Sartori charakterisierte es als Prototyp eines „polarisierten Pluralismus" 55 , der mit Recht als Wurzel der geringen Regierungsstabilität gelten kann. Zu Anfang der 1990er Jahre erfuhr dieses Parteiensystem einen tiefgreifenden Einschnitt. Ob das Ende des Wandels in einem bipolaren Parteiensystem mit höherer Regierungsstabilität oder einer Rückkehr zu den Verhältnissen der Ersten Republik liegt, ist dabei noch immer nicht endgültig abzusehen. ${ }^{56}$

Sind starke Zweite Kammern mit eigenständiger Funktion generell unvereinbar mit der parlamentarischen Demokratie? Arend Lijphart sieht übergroße Koalitionen als einen konsensusdemokratischen Weg an, um zu verhindern, dass ein starker Bikameralismus die Regierungsstabilität in parlamentarischen Demokratien beeinträchtigt. ${ }^{57}$ Damit würde aber nur eine Quelle der Regierungsinstabilität durch eine andere ersetzt. Es dürfte nämlich folgende Faustregel gelten: Je größer und heterogener eine Regierungskoalition ist, desto schwieriger fällt es, sie aufrechtzuerhalten. ${ }^{58}$ Sartori verficht folgende Ansicht: Je asymme-

55 Mit (a) mindestens fünf Parteien, (b) keiner klaren Mehrheit für eine Partei, (c) starken AntiSystemparteien und (d) einer großen Uneinigkeit der übrigen Parteien über wesentliche Themen. Vgl. dazu Giovanni Sartori, European Political Parties: The Case of Polarized Pluralism, in: Joseph LaPalombara / Myron Weiner (Hrsg.), Political Parties and Political Development, Princeton 1966, S. 137 - 176; ders., Teoria dei partiti e caso Italiano, Mailand 1983.

56 Vgl. unter anderem Martin Bull / Martin Rhodes (Hrsg.), Crisis and Transition in Italian Politics, London 1997; Elisabeth Fix, Italiens Parteiensystem im Wandel. Von der Ersten zur Zweiten Republik, Frankfurt am Main / New York 1999; Mario Caciagli, Die dramatische Umwandlung und das ungewisse Schicksal des italienischen Parteiensystems, in: Wolfgang Merkel / Andreas Busch (Hrsg.), Demokratie in Ost und West, Frankfurt am Main 1999, S. 455 - 474; Reimut Zohlnhöfer, Das italienische Parteiensystem nach den Wahlen: Stabilisierung des fragmentierten Bipolarismus oder Rückkehr zur „ersten Republik“?, in: ZParl, 33. Jg. (2002), H. 2, S. 271 -290 .

57 Vgl. Arend Lijphart, Democracies. Patterns of Majoritarian and Consensus Government in Twenty-One Countries, New Haven / London 1984, S. 104.

58 Institutionell ließe sich die Problematik des Bikameralismus für die Regierungsstabilität in parlamentarischen Demokratien dabei leicht mit folgender Regelung lösen: Die Regierung stürzt nur, wenn ihr beide Kammern das Misstrauen aussprechen. So gestaltete symmetrische Zweikammersysteme in parlamentarischen Demokratien würden die Regierungsstabilität nicht senken, sondern erhöhen. Es ist eine ganz andere Frage, ob eine solche Regelung wünschenswert ist. Diese institutionelle Anlage findet sich derzeit nur in der elektoralen Autokratie Haiti, das nicht zu den Untersuchungsfällen dieser Studie gehört. Vgl. zu diesem Fall die kurzen Anmerkungen in 
trischer zwei Kammern sind, desto mehr kann sich ihre Zusammensetzung unterscheiden. Je symmetrischer jedoch zwei Kammern sind, desto weniger verschieden sollten sie zusammengesetzt sein. ${ }^{59}$ Nun dürfte zwar für parlamentarische Demokratien, die dem zweiten Ratschlag folgen, die Regierungsstabilität tatsächlich steigen. Zugleich provoziert aber eine solche Zweite Kammer die Frage, wozu der Wähler in einer Demokratie zwei gleich starke Organe auf die gleiche Weise wählen soll, die auch noch den gleichen Zweck haben. Wenn man auf den „Abkühlungseffekt" nicht verzichten will, kann man schließlich auch wie in Norwegen die Nationalversammlung nach der Wahl in zwei Kammern teilen. Was bleibt, ist folgende Aussage: Während starker Bikameralismus und Präsidentialismus in die gleiche Richtung wirken, stehen starker Bikameralismus und Parlamentarismus zwangsläufig in einem Spannungsverhältnis zueinander. Hier gelten im Kern dieselben Argumente wie jene, die - fokussiert auf das Spannungsverhältnis von Parteiendemokratie und Föderalismus Gerhard Lehmbruch in Deutschland ausgeführt hat. ${ }^{60}$ Von den Ursprüngen des Parlamentarismus in Großbritannien und des Präsidentialismus in den USA her gilt nämlich beim Blick auf die „separation of powers“: „Parliamentarism spells majoritarianism“ und „Presidentialism spells consensualism" 61 .

Eine These, die sich aus der Präsidentialismuskritik von Juan Linz und anderen ${ }^{62}$ ableiten lässt, lautet: Je größer die Wahrscheinlichkeit von institutionellen Blockaden in einer Regierungsform, desto niedriger dürften die durchschnittlichen politischen Leistungen ausfallen. Demnach liegen die Probleme des Präsidentialismus gerade nicht daran, dass der Präsidentialismus mehrheitsdemokratisch organisiert ist. Scott Mainwaring und Matthew Shugart haben auf diese Kritik am Präsidentialismus gekontert, dass mit Blick auf symmetrische Zweikammersysteme auch in parlamentarischen Demokratien die Gefahr institutioneller Blockaden bestehe. ${ }^{63}$ Die hier angestellte Untersuchung führt allerdings zu folgendem Ergebnis: Mit den starken und stark legitimierten Zweiten Kammern steht dem Regierungschef in präsidentiellen Demokratien häufiger als in parlamentarischen ein zusätzlicher mächtiger institutioneller Vetopunkt gegenüber. Daher scheint die Gefahr einer politischen Blockade durch eine starke Zweite Kammer in präsidentiellen Demokratien größer als in parlamentarischen. Für alle politischen Akteure, vor allem aber für den Präsidenten, birgt ein symmetrisches Zweikammersystem ein erhebliches Risiko der Frustration. Theoretisch kann ein populärer, nicht parteigebundener politischer Außenseiter zum Präsidenten werden, in der Ersten Kammer Partei A eine Mehrheit haben und in der Zweiten

Matthew Søberg Shugart / John M. Carey, Presidents and Assemblies: Constitutional Design and Electoral Dynamics, Cambridge 1992, S. 73.

59 Vgl. Giovanni Sartori, Comparative Constitutional Engineering: An Inquiry into Structures, Incentives and Outcomes, 2. Auflage, New York 1997, S. 188.

60 Vgl. Gerhard Lehmbruch, Parteienwettbewerb im Bundesstaat: Regelsysteme und Spannungslagen im politischen System der Bundesrepublik Deutschland, 3. aktualisierte und erweiterte Auflage, Opladen 1997.

61 Auf der Ebene der neuen Machtteilung, der Teilung der Macht zwischen Parteien, sieht dies allerdings anders aus. So gibt es in präsidentiellen Demokratien etwa deutlich weniger Koalitionsregierungen als in parlamentarischen (Nichtwestminster-)Demokratien. Vgl. zu den empirischen Zahlen Steffen Kailitz, a.a.O.

62 Vgl. unter anderem Juan Linz, a.a.O., S. 3 - 87.

63 Vgl. Scott Mainwaring / Matthew Søberg Shugart, Juan Linz, Presidentialism and Democracy: A Critical Appraisal, o.O. o.J. (Notre Dame 1993), S. 8 f. 
Kammer Partei B vorherrschen. ${ }^{64}$ Effektives Regieren wäre - unter der Prämisse, dass die Parteien in beiden Kammern diszipliniert abstimmen - kaum möglich und die Demokratiestabilität gefährdet. Bei dieser Argumentation ist zu bedenken, dass die „Federalists“ gerade eine Regierungsform konstruieren wollten, in der, erstens, das Parlament und nicht die Regierung die Gesetzgebungsagenda bestimmt und, zweitens, die Mitglieder der Ersten und Zweiten Kammer aufgrund persönlicher Präferenzen abstimmen und keine Parteidisziplin herrscht, die die institutionelle Gewaltenteilung aushöhlt. Die Probleme entstehen also erst dadurch, dass diese beiden Prämissen vor allem aufgrund der zunehmenden Komplexität der Staatsaufgaben nicht mehr haltbar sind.

Wenn die „separation of power" nicht mit einer „separation of purpose“ einhergeht, macht dies unterschiedliche Mehrheitsverhältnisse unwahrscheinlicher. Damit sinkt die Gefahr, dass sich beide Kammern gegenseitig blockieren. ${ }^{65}$ Wenn beide Kammern einen klar unterscheidbaren Zweck verfolgen (zum Beispiel nationale vs. territoriale Interessen) steigt dagegen die Möglichkeit unterschiedlicher Mehrheiten und der Politikstabilität (da Reformen so schwerer durchführbar sind). In dieser Hinsicht erhöht die stärkere eigenständige Legitimation Zweiter Kammern in präsidentiellen Demokratien neutral betrachtet wie von den Schöpfern gewünscht - die Wahrscheinlichkeit der Policy-Stabilität; kritisch gewendet macht sie einen „Reformstau“ wahrscheinlicher.

Möglicherweise sind von einem starken Bikameralismus auch nicht nur Folgen auf die Policy-Stabilität, sondern auch auf die Politikrichtung zu erwarten. So kam Adrian Vatter nach einer Untersuchung der parteipolitischen Repräsentationsunterschiede zwischen Erster und Zweiter Kammer in den OECD-Staaten zu dem Schluss, dass in der Zweiten Kammer liberale und konservative Parteien deutlich stärker seien als in der Ersten Kammer. Dadurch dürften „politische Systeme mit einer starken Zweiten Kammer signifikant liberalere (weniger Staat) als auch vermehrt konservative (status quo) Politikresultate liefern "66. Wenn dies auch für unser weit umfangreicheres Untersuchungsfeld gelten würde, ergäbe sich daraus der Schluss, dass durch den starken Bikameralismus in präsidentiellen Demokratien nicht nur die Policy-Stabilität, sondern auch die Policy-Richtung beeinflusst würde. Der Blick auf die USA stützt die stärkere Prägung durch liberales und konservatives Gedankengut impressionistisch, kann aber eine systematische Studie zu dieser Frage nicht ersetzen.

Im Kern ist unbestreitbar, dass die Zweiten Kammern in zahlreichen präsidentiellen Demokratien einen starken zusätzlichen Vetopunkt darstellen. So sind Fälle beobachtbar, in denen Programme des Regierungschefs durch eine starke Zweite Kammer in ihrer Durchsetzung behindert werden. Ein Beispiel für anhaltende Abstimmungsprobleme zwischen Erster und Zweiter Kammer sind die Philippinen. ${ }^{67}$ In dieser präsidentiellen Demokratie ist nämlich kein Ende für das Navetteverfahren vorgesehen.

Ein Blick auf die Gesamtheit der gegenwärtigen präsidentiellen Demokratien macht aber eine gewichtige Einschränkung notwendig: Eine starke Zweite Kammer ist - anders

64 Empirisch ist dieses Schlimmster-Fall-Szenario aber in den präsidentiellen Demokratien sehr selten.

65 Vgl. dazu Gary W. Cox / Matthew D. McCubbins, a.a.O., S. 21 - 63.

66 Adrian Vatter, a.a.O., S. 127.

67 Vgl. Aurel Croissant, Von der Transition zur defekten Demokratie: demokratische Entwicklung in den Philippinen, Südkorea und Thailand, Wiesbaden 2002, S. 165. 
als George Tsebelis' Vetospieleransatz oder der Vetopunktansatz von André Kaiser nahe legen $^{68}$, und zwar unabhängig von der parteipolitischen Kräfteverteilung - nämlich nicht zwangsläufig ein zusätzlicher institutioneller Vetospieler oder Vetopunkt. So sind zwei Kammern zwar gleich stark, aber kein zusätzlicher Vetopunkt für die Regierung, wenn am Ende des Gesetzgebungsverfahrens jene Kammer entscheidet, in der das Gesetz zuerst vorgelegt wurde (wie in Argentinien, Paraguay, Chile). Obgleich die Kammern gleich stark sind, haben beide bei dieser Konstellation nur eine geringe Vetomacht, politische Initiativen einer Mehrheit der anderen Kammer zu blockieren. In Chile bringt die Verfassung den Präsidenten gar in die Lage, dass die zwei gleich starken Parlamentskammern nicht zwei Vetospieler sind. Verabschiedet nämlich eine Kammer den Gesetzesvorschlag des Präsidenten, kann die andere Kammer nur noch mit einer Dreiviertelmehrheit der anwesenden Parlamentarier verhindern, dass der Präsident seinen Entwurf durchsetzt. Das starke Zweikammersystem wirkt in dieser speziellen Konfiguration also in der politischen Praxis, so paradox dies anmuten mag, als ein Instrument zugunsten der Machtkonzentration beim Präsidenten und nicht in Richtung einer Machtteilung. Es lässt sich daher nicht in allen Fällen behaupten: „Strong bicameralism spells consensual democracy. “69

Allerdings ist die parlamentarische Demokratie Deutschlands geradezu ein Musterbeispiel für diese Argumentation Lijpharts. Hier wie etwa auch in der Schweiz ist die starke Zweite Kammer ein klar konsensusdemokratisches Element. Der relativ starke deutsche Bundesrat gehört dabei unter den parlamentarischen Regierungssystemen - zusammen mit Australien, Indien, Spanien und Südafrika - zur Minderheit der Zweiten Kammern, die über eine für die moderne Demokratie bedeutsame eigenständige Funktion verfügen, nämlich die Repräsentation subnationaler Interessen auf bundesstaatlicher Ebene. Die Abstimmungen im Bundesrat werden allerdings in der Praxis häufiger durch die Parteilinie der Mitglieder als durch die Landeszugehörigkeit bestimmt. ${ }^{70}$ Konkrete Länderinteressen sind bei vielen konkreten Abstimmungen im Bundesrat allerdings auch gar nicht recht zu definieren (zum Beispiel in der Sozial- oder Gesundheitspolitik). Ob in Deutschland der von den „Federalists“ angeführte Vorteil einer Zweiten Kammer (bessere Politik durch Verhinderung übereilter Gesetzgebungsvorhaben) überwiegt und der Bundesrat eher noch gestärkt werden sollte, oder ob doch der etwa von Walter Bagehot genannte Nachteil (schlechtere Politik durch gegenseitige Blockade der Parlamentskammern) schwerer wiegt und der Bundesrat entscheidend geschwächt oder gar abgeschafft werden sollte, ist und bleibt eine Glaubensfrage.

68 Vgl. George Tsebelis, Veto Players: How Political Institutions Work, Princeton 2002; André Kaiser, Institutional Regimes, in: Jan van Deth (Hrsg.), Comparative Politics. The Problem of Equivalence, London 1998, S. 213. Speziell mit Blick auf den Bikameralismus siehe George Tsebelis / Jeanette Money, a.a.O., S. 75 f.

69 Diese Folgerung ist die Sentenz aus: Arend Lijphart, a.a.O. (Fn. 5), S. $200-215$.

70 Vgl. zu diesem Aspekt Sven Leunig, Länder- versus Parteiinteressen im Bundesrat - Realer Dualismus oder fiktive Differenzierung?, in: APuZ, B 50 / 51 (2004), S. 33 - 38; Gerd Strohmeier, Der Bundesrat: Vertretung der Länder oder Instrument der Parteien?, in: ZParl, 35. Jg. (2004), H. 4, S. $717-731$. 


\section{Anhang}

\section{Ein- und Zweikammersysteme in den Demokratien des Jahres 2007}

\begin{tabular}{|c|c|c|c|c|}
\hline \multicolumn{5}{|c|}{ parlamentarische Demokratien } \\
\hline & $\begin{array}{c}\text { Existenz Zweite } \\
\text { Kammer } \\
\end{array}$ & Stärke & $\begin{array}{c}\text { demokratische } \\
\text { Legitimation }\end{array}$ & $\begin{array}{l}\text { eigenständige } \\
\text { Funktion } \\
\end{array}$ \\
\hline Albanien & 0 & & & \\
\hline Australien & 1 & 66,6 & 100 & 100 \\
\hline Bahamas & 1 & 33,3 & 50 & 0 \\
\hline Bangladesch & 0 & & & \\
\hline Barbados & 1 & 33,3 & 50 & 0 \\
\hline Belgien & 1 & 66,6 & 75 & 75 \\
\hline Belize & 1 & 33,3 & 50 & 0 \\
\hline Botswana & 0 & & & \\
\hline Dänemark & 0 & & & \\
\hline Deutschland & 1 & 66,6 & 75 & 100 \\
\hline Estland & 0 & & & \\
\hline Fiji & 1 & 33,3 & 25 & 0 \\
\hline Großbritannien & 1 & 33,3 & 50 & 0 \\
\hline Indien & 1 & 33,3 & 75 & 100 \\
\hline Israel & 0 & & & \\
\hline Italien & 1 & 100 & 100 & 0 \\
\hline Jamaika & 1 & 33,3 & 50 & 0 \\
\hline Japan & 1 & 66,6 & 100 & 0 \\
\hline Kanada & 1 & 33,3 & 50 & 75 \\
\hline Lettland & 0 & & & \\
\hline Luxemburg & 0 & & & \\
\hline Malta & 0 & & & \\
\hline Mauritius & 0 & & & \\
\hline Neuseeland & 0 & & & \\
\hline Niederlande & 1 & 66,6 & 75 & 0 \\
\hline Norwegen & 0 & & & \\
\hline Papua Neuguinea & 0 & & & \\
\hline Trinidad und Tobago & 1 & 66,6 & 50 & 0 \\
\hline Salomonen & 0 & & & \\
\hline Schweden & 0 & & & \\
\hline Spanien & 1 & 66,6 & 87,5 & 100 \\
\hline Südafrika & 1 & 66,6 & 75 & 100 \\
\hline Thailand & 0 & & & \\
\hline Tschechien & 1 & 66,6 & 100 & 0 \\
\hline Türkei & 1 & 33,3 & 25 & 0 \\
\hline Ungarn & 0 & & & \\
\hline
\end{tabular}




\begin{tabular}{|c|c|c|c|c|}
\hline \multicolumn{5}{|c|}{ quasi-parlamentarische Demokratien } \\
\hline & \begin{tabular}{|c|} 
Existenz Zweite \\
Kammer
\end{tabular} & Stärke & $\begin{array}{l}\text { demokratische } \\
\text { Legitimation }\end{array}$ & $\begin{array}{l}\text { eigenständige } \\
\text { Funktion }\end{array}$ \\
\hline Bulgarien & 0 & & & \\
\hline Finnland & 0 & & & \\
\hline Griechenland & 0 & & & \\
\hline Irland & 1 & 33,3 & 62,5 & 25 \\
\hline Island & 0 & & & \\
\hline Litauen & 0 & & & \\
\hline Mazedonien & 0 & & & \\
\hline Österreich & 1 & 66,6 & 75 & 100 \\
\hline Portugal & 0 & & & \\
\hline Slowakei & 0 & & & \\
\hline Slowenien & 1 & 33,3 & 62,5 & 50 \\
\hline \multicolumn{5}{|c|}{ duale Exekutive } \\
\hline & \begin{tabular}{|c|} 
Existenz Zweite \\
Kammer
\end{tabular} & Stärke & $\begin{array}{l}\text { demokratische } \\
\text { Legitimation }\end{array}$ & $\begin{array}{l}\text { eigenständige } \\
\text { Funktion }\end{array}$ \\
\hline Frankreich & 1 & 33,3 & 75 & 75 \\
\hline Kap Verde & 0 & & & \\
\hline Moldawien & 0 & & & \\
\hline Mongolei & 0 & & & \\
\hline Polen & 1 & 33,3 & 100 & 0 \\
\hline Rumänien & 1 & 100 & 100 & 0 \\
\hline Taiwan & 1 & 66,6 & 50 & 0 \\
\hline Ukraine & 0 & & & \\
\hline \multicolumn{5}{|c|}{ quasi-präsidentielle Demokratien } \\
\hline & \begin{tabular}{|c|} 
Existenz Zweite \\
Kammer \\
\end{tabular} & Stärke & $\begin{array}{l}\text { demokratische } \\
\text { Legitimation }\end{array}$ & $\begin{array}{l}\text { eigenständige } \\
\text { Funktion }\end{array}$ \\
\hline Armenien & 0 & & & \\
\hline Guyana & 0 & & & \\
\hline Madagaskar & 1 & 33,3 & 62,5 & 75 \\
\hline Mali & 0 & & & \\
\hline Namibia & 1 & 66,6 & 75 & 100 \\
\hline Peru & 0 & & & \\
\hline Russland & 1 & 66,6 & 75 & 100 \\
\hline Senegal & 0 & & & \\
\hline Sri Lanka & 0 & & & \\
\hline Südkorea & 0 & & & \\
\hline
\end{tabular}




\begin{tabular}{|c|c|c|c|c|}
\hline \multicolumn{5}{|c|}{ präsidentielle Demokratien } \\
\hline & $\begin{array}{c}\text { Existenz Zweite } \\
\text { Kammer } \\
\end{array}$ & Stärke & $\begin{array}{l}\text { demokratische } \\
\text { Legitimation }\end{array}$ & $\begin{array}{l}\text { eigenständige } \\
\text { Funktion }\end{array}$ \\
\hline Argentinien & 1 & 100 & 100 & 100 \\
\hline Benin & 0 & & & \\
\hline Bolivien & 1 & 100 & 100 & 100 \\
\hline Brasilien & 1 & 100 & 100 & 100 \\
\hline Chile & 1 & 100 & 100 & 100 \\
\hline Costa Rica & 0 & & & \\
\hline Dominikanische Republik & 1 & 100 & 100 & 100 \\
\hline Ecuador & 0 & & & \\
\hline El Salvador & 0 & & & \\
\hline Guatemala & 0 & & & \\
\hline Honduras & 0 & & & \\
\hline Kolumbien & 1 & 100 & 100 & 75 \\
\hline Malawi & 0 & & & \\
\hline Mexiko & 1 & 100 & 100 & 100 \\
\hline Nicaragua & 0 & & & \\
\hline Panama & 0 & & & \\
\hline Paraguay & 1 & 100 & 100 & 75 \\
\hline Philippinen & 1 & 100 & 100 & 0 \\
\hline Surinam & 0 & & & \\
\hline Uruguay & 1 & 100 & 100 & 100 \\
\hline USA & 1 & 100 & 100 & 100 \\
\hline Venezuela & 0 & & & \\
\hline Zypern - griechischer Teil & 0 & & & \\
\hline \multicolumn{5}{|c|}{ keiner Regierungsform zugeordneter Fall } \\
\hline Schweiz & 1 & 100 & 100 & 100 \\
\hline
\end{tabular}

